\title{
Multiscale techniques for parabolic equations
}

\author{
Axel Målqvist ${ }^{1} \cdot$ Anna Persson $^{1}$
}

Received: 30 April 2015 / Revised: 19 October 2016 / Published online: 20 July 2017

(C) The Author(s) 2017. This article is an open access publication

\begin{abstract}
We use the local orthogonal decomposition technique introduced in Målqvist and Peterseim (Math Comput 83(290):2583-2603, 2014) to derive a generalized finite element method for linear and semilinear parabolic equations with spatial multiscale coefficients. We consider nonsmooth initial data and a backward Euler scheme for the temporal discretization. Optimal order convergence rate, depending only on the contrast, but not on the variations of the coefficients, is proven in the $L_{\infty}\left(L_{2}\right)$-norm. We present numerical examples, which confirm our theoretical findings.
\end{abstract}

Mathematics Subject Classification 35K05 - 35K58 - 65M60

\section{Introduction}

In this paper we study numerical solutions to parabolic equations with highly varying coefficients. These equations appear, for instance, when modeling physical behavior in a composite material or a porous medium. Such problems are often referred to as multiscale problems.

Axel Målqvist's work is supported by the Swedish Research Council.

$\triangle \quad$ Anna Persson

peanna@chalmers.se

Axel Målqvist

axel@chalmers.se

1 Department of Mathematical Sciences, Chalmers University of Technology and University of Gothenburg, Göteborg, Sweden 
Convergence of optimal order of classical finite element methods (FEMs) based on continuous piecewise polynomials relies on at least spatial $H^{2}$-regularity. More precisely, for piecewise linear polynomials, the error bound depends on $\|u\|_{H^{2}}$, which may be proportional to $\epsilon^{-1}$ if the diffusion coefficient varies on a scale of $\epsilon$. Thus, the mesh width $h$ typically must fulfill $h<\epsilon$ to achieve convergence. However, this is not computationally feasible in many applications. To overcome this issue, several numerical methods have been proposed, see, for example, [2,8,13,15,16,19], and references therein. In particular, $[15,16]$ consider linear parabolic equations.

In [13] a generalized finite element method (GFEM) was introduced and convergence of optimal order was proven for elliptic multiscale equations. The method builds on ideas from the variational multiscale method $[8,10]$, which is based on a decomposition of the solution space into a (coarse) finite dimensional space and a residual space for the fine scales. The method in [13], often referred to as local orthogonal decomposition, constructs a generalized finite element space where the basis functions contain information from the diffusion coefficient and have support on small vertex patches. With this approach, convergence of optimal order can be proved for an arbitrary positive and bounded diffusion coefficient. Restrictive assumptions such as periodicity of the coefficients or scale separation are not needed. Some recent works $[1,6,7,14]$ show how this method can be applied to boundary value problems, eigenvalue problems, semilinear elliptic equations, and linear wave equations.

In this paper we apply the technique introduced in [13] to parabolic equations with multiscale coefficients. We use the diffusion coefficient to construct a generalized finite element space and for the discretization of the temporal domain we use the backward Euler scheme. Using tools from classical finite element theory for parabolic equations, see, e.g, $[11,12,18]$, and references therein, we prove convergence of optimal order in the $L_{\infty}\left(L_{2}\right)$-norm for linear and semilinear equations under minimal regularity assumptions and nonsmooth initial data. The analysis is completed with numerical examples that support our theoretical findings.

In Sect. 2 we describe the problem formulation and the assumptions needed to achieve sufficient regularity of the solution. Section 3 describes the numerical approximation and presents the resulting GFEM. In Sect. 4 we prove error bounds and in Sect. 5 we extend the results to semilinear parabolic equations. Finally, in Sect. 6 we present some numerical examples.

\section{Problem formulation}

We consider the parabolic problem

$$
\begin{array}{ll}
c \dot{u}-\nabla \cdot(A \nabla u)=f, & \text { in } \Omega \times(0, T], \\
u=0, & \text { on } \partial \Omega \times(0, T], \\
u(\cdot, 0)=u_{0}, & \text { in } \Omega,
\end{array}
$$

where $T>0$ and $\Omega$ is a bounded polygonal/polyhedral domain in $\mathbb{R}^{d}, d \leq 3$. We assume $c=c(x), A=A(x)$, and $f=f(x, t)$. Here we allow both $c$ and $A$ to be multiscale (in space), but independent of the time variable. 
We let $H^{1}(\Omega)$ denote the classical Sobolev space with norm

$$
\|v\|_{H^{1}(\Omega)}^{2}=\|v\|_{L_{2}(\Omega)}^{2}+\|\nabla v\|_{L_{2}(\Omega)}^{2}
$$

and $V=H_{0}^{1}(\Omega)$ the space of functions in $H^{1}(\Omega)$ that vanishes on $\partial \Omega$. We use $H^{-1}(\Omega)$ to denote the dual space to $V$. Furthermore, we use the notation $L_{p}(0, T ; X)$ for the Bochner space with finite norm

$$
\begin{aligned}
\|v\|_{L_{p}(0, T ; X)} & =\left(\int_{0}^{T}\|v\|_{X}^{p} \mathrm{dt}\right)^{1 / p}, \quad 1 \leq p<\infty, \\
\|v\|_{L_{\infty}(0, T ; X)} & =\operatorname{ess} \sup _{0 \leq t \leq T}\|v\|_{X}
\end{aligned}
$$

where $X$ is a Banach space equipped with norm $\|\cdot\|_{X}$. Here $v \in H^{1}(0, T ; X)$ means $v, \dot{v} \in L_{2}(0, T ; X)$. The dependence on the interval $[0, T]$ and the domain $\Omega$ is frequently suppressed and we write, for instance, $L_{2}\left(L_{2}\right)$ for $L_{2}\left(0, T ; L_{2}(\Omega)\right)$. Finally, we abbreviate the $L_{2}$-norm $\|\cdot\|:=\|\cdot\|_{L_{2}(\Omega)}$ and define $\|\cdot\|\|:=\| A^{1 / 2} \nabla \cdot \|$.

To ensure existence, uniqueness, and sufficient regularity, we make the following assumptions on the data.

We assume

(A1) $A \in L_{\infty}\left(\Omega, \mathbb{R}^{d \times d}\right)$, symmetric, and

$$
\begin{gathered}
0<\alpha_{1}:=\operatorname{essinf}_{x \in \Omega} \inf _{v \in \mathbb{R}^{d} \backslash\{0\}} \frac{A(x) v \cdot v}{v \cdot v}, \\
\infty>\alpha_{2}:=\operatorname{ess} \sup _{x \in \Omega} \sup _{v \in \mathbb{R}^{d} \backslash\{0\}} \frac{A(x) v \cdot v}{v \cdot v},
\end{gathered}
$$

(A2) $c \in L_{\infty}(\Omega, \mathbb{R})$ and

$$
0<\gamma_{1}:=\operatorname{essinf}_{x \in \Omega} c(x) \leq \operatorname{ess}_{\sup } \in \Omega(x)=: \gamma_{2}<\infty,
$$

(A3) $u_{0} \in L_{2}$,

(A4) $f, \dot{f} \in L_{\infty}\left(L_{2}\right)$.

We let $(u, v)=\int_{\Omega} u v$ denote the classical $L_{2}$ inner product and define

$$
(\cdot, \cdot)_{c}:=(c \cdot, \cdot) .
$$

Due to (A2) this is an inner product and the induced norm $\left\|c^{1 / 2} \cdot\right\|$ is equivalent to the classical $L_{2}$-norm.

We emphasize that throughout this work $C$ denotes a constant that may depend on the bounds $\alpha_{1}$ and $\alpha_{2}$ (often through the contrast $\alpha_{2} / \alpha_{1}$ ), the bounds $\gamma_{1}$ and $\gamma_{2}$, the shape regularity parameter $\varrho$ (3.1) of the mesh, the final time $T$, and the size of the domain $\Omega$, but not on the mesh size parameters nor the derivatives of the coefficients in $A$ or $c$. The fact that the constant does not depend on the derivatives of $A$ nor $c$ is crucial, since these (if they exist) are large for the problems of interest. This is sometimes also noted as $C$ being independent of the variations of $A$ and $c$.

We now formulate the variational form of problem (2.1). Find $u(\cdot, t) \in V$ such that $u(\cdot, 0)=u_{0}$ and 


$$
(\dot{u}, v)_{c}+a(u, v)=(f, v), \quad \forall v \in V, t \in(0, T],
$$

and $a(u, v)=(A \nabla u, \nabla v)$.

The following theorem states existence and uniqueness of a solution to (2.2). The proof is based on Galerkin approximations, see, e.g., [5,9].

Theorem 2.1 Assume that (A1)-(A4) are satisfied. Then there exists a unique solution $u$ to (2.2) such that $u \in L_{2}\left(0, T ; H_{0}^{1}\right)$ and $\dot{u} \in L_{2}\left(0, T ; H^{-1}\right)$.

\section{Numerical approximation}

In this section we describe the local orthogonal decomposition method presented in [13] to define a generalized finite element method for the multiscale problem (2.2).

First we introduce some notation. Let $\left\{\mathcal{T}_{h}\right\}_{h>0}$ and $\left\{\mathcal{T}_{H}\right\}_{H>h}$ be families of shape regular triangulations of $\Omega$ where $h_{K}:=\operatorname{diam}(K)$, for $K \in \mathcal{T}_{h}$, and $H_{K}:=\operatorname{diam}(K)$, for $K \in \mathcal{T}_{H}$. We also define $H:=\max _{K \in \mathcal{T}_{H}} H_{K}$ and $h:=\max _{K \in \mathcal{T}_{h}} h_{K}$. Furthermore, we let $\varrho>0$ denote the shape regularity parameter of the mesh $\mathcal{T}_{H}$;

$$
\varrho:=\max _{K \in \mathcal{T}_{H}} \varrho_{K}, \text { with } \varrho_{K}:=\frac{\operatorname{diam} B_{K}}{\operatorname{diam} K}, \text { for } K \in \mathcal{T}_{H},
$$

where $B_{K}$ is the largest ball contained in $K$.

Now define the classical piecewise affine finite element spaces

$$
\begin{aligned}
V_{H} & =\left\{v \in C(\bar{\Omega}): v=0 \text { on } \partial \Omega,\left.v\right|_{K} \text { is a polynomial of degree } \leq 1, \forall K \in \mathcal{T}_{H}\right\}, \\
V_{h} & =\left\{v \in C(\bar{\Omega}): v=0 \text { on } \partial \Omega,\left.v\right|_{K} \text { is a polynomial of degree } \leq 1, \forall K \in \mathcal{T}_{h}\right\} .
\end{aligned}
$$

We let $\mathcal{N}$ denote the interior nodes of $V_{H}$ and $\varphi_{x}$ the corresponding hat function for $x \in \mathcal{N}$, such that $\operatorname{span}\left(\left\{\varphi_{x}\right\}_{x \in \mathcal{N}}\right)=V_{H}$. We further assume that $\mathcal{T}_{h}$ is a refinement of $\mathcal{T}_{H}$, so that $V_{H} \subseteq V_{h}$. Finally, we also need the finite element mesh $\mathcal{T}_{H}$ of $\Omega$ to be such that the $L_{2}$-projection $P_{H}$ onto the finite element space $V_{H}$ is stable in $H^{1}$-norm, see, e.g., [3], and the references therein.

To discretize in time we introduce the uniform discretization

$$
0=t_{0}<t_{1}<\cdots<t_{N}=T, \quad \text { where } t_{n}-t_{n-1}=\tau
$$

Let $U_{n}$ be the approximation of $u(t)$ at time $t=t_{n}$ and denote $f_{n}:=f\left(t_{n}\right)$. Using the notation $\bar{\partial}_{t} U_{n}=\left(U_{n}-U_{n-1}\right) / \tau$ we now formulate the classical backward Euler FEM; find $U_{n} \in V_{h}$ such that

$$
\left(\bar{\partial}_{t} U_{n}, v\right)_{c}+a\left(U_{n}, v\right)=\left(f_{n}, v\right), \quad \forall v \in V_{h},
$$

for $n=1, \ldots, N$ and $U_{0} \in V_{h}$ is some approximation of $u_{0}$. We also define the operator $\mathcal{A}_{h}: V_{h} \rightarrow V_{h}$ by

$$
\left(\mathcal{A}_{h} v, w\right)=a(v, w), \quad \forall v, w \in V_{h}
$$


The convergence of the classical finite element approximation (3.3) depends on $\left\|D^{2} u\right\|$, where $D^{2}$ denotes the second order derivatives. If the diffusion coefficient $A$ oscillates on a scale of $\epsilon$ we may have $\left\|D^{2} u\right\| \sim \epsilon^{-1}$, see [17] for a further discussion. The total error is thus typically bounded by $\left\|u\left(t_{n}\right)-U_{n}\right\| \leq C\left(\tau+(h / \epsilon)^{2}\right)$, which is small only if $h<\epsilon$.

The purpose of the method described in this paper is to find an approximate solution, let us denote it by $\hat{U}$ for now, in some space $\hat{V} \subset V_{h}$, such that $\operatorname{dim} \hat{V}=\operatorname{dim} V_{H}$, for $H>h$, and the error $\left\|U_{n}-\hat{U}_{n}\right\| \leq C H^{2}$. Here $C$ is independent of the variations in $A$ and $c$ and $\hat{U}_{n}$ is less expensive to compute than $U_{n}$. The total error is then the sum of two terms

$$
\left\|u\left(t_{n}\right)-\hat{U}_{n}\right\| \leq\left\|u\left(t_{n}\right)-U_{n}\right\|+\left\|U_{n}-\hat{U}_{n}\right\|,
$$

where the first term is the error due to the classical FEM approximation with backward Euler discretization in time. This is small if $h$ (and $\tau$ ) is chosen sufficiently small, that is, if $h$ resolves the variations of $A$. Hence, we think of $h>0$ as fixed and appropriately chosen. Our aim is now to analyze the error $\left\|U_{n}-\hat{U}_{n}\right\|$.

We emphasize that $\hat{V}=V_{H}$ is not sufficient. The total error would in this case typically be $\left\|u\left(t_{n}\right)-\hat{U}_{n}\right\| \sim\left(\tau+(H / \epsilon)^{2}\right)$, which is small only if $H<\epsilon$.

The next theorem states some regularity results for (3.3).

Theorem 3.1 Assume that (A1)-(A4) are satisfied. Then, for $1 \leq n \leq N$, there exists a unique solution $U_{n}$ to (3.3) such that $U_{n} \in V_{h}$. Furthermore, if $U_{0}=0$, then we have the bound

$$
\left\|\bar{\partial}_{t} U_{n}\right\| \leq C\left(\|f\|_{L_{\infty}\left(L_{2}\right)}+\|\dot{f}\|_{L_{\infty}\left(L_{2}\right)}\right),
$$

and, if $f=0$, then

$$
\left\|\bar{\partial}_{t} U_{n}\right\| \leq C t_{n}^{-1}\left\|U_{0}\right\|, n \geq 1, \quad\left\|\bar{\partial}_{t} \bar{\partial}_{t} U_{n}\right\| \leq C t_{n}^{-2}\left\|U_{0}\right\|, n \geq 2,
$$

where $C$ depends on $\alpha_{1}, \gamma_{1}, \gamma_{2}$ and $T$, but not on the variations of $A$ or $c$.

Proof From (3.3) it follows for $n \geq 2$ that

$$
\left(\bar{\partial}_{t} \bar{\partial}_{t} U_{n}, v\right)_{c}+a\left(\bar{\partial}_{t} U_{n}, v\right)=\left(\bar{\partial}_{t} f_{n}, v\right), \quad \forall v \in V_{h},
$$

and choosing $v=\bar{\partial}_{t} U_{n}$ we derive

$$
\left\|c^{1 / 2} \bar{\partial}_{t} U_{n}\right\| \leq\left\|c^{1 / 2} \bar{\partial}_{t} U_{1}\right\|+\frac{1}{\sqrt{\gamma_{1}}} \sum_{j=2}^{n} \tau\left\|\bar{\partial}_{t} f_{j}\right\| .
$$

From (3.3) we have, since $U_{0}=0,\left\|c^{1 / 2} \bar{\partial}_{t} U_{1}\right\| \leq \gamma_{1}^{-1 / 2}\left\|f_{1}\right\|$. Finally, using the inequality

$$
\sum_{j=2}^{n} \tau\left\|\bar{\partial}_{t} f_{j}\right\| \leq \sum_{j=2}^{n} \max _{t_{j-1} \leq \xi \leq t_{j}} \tau\|\dot{f}(\xi)\| \leq C\|\dot{f}\|_{L_{\infty}\left(L_{2}\right)}
$$

and the bounds on $c$ in (A2) we deduce (3.5). 
To derive the bounds in (3.6), we define the solution operator $E_{n}$ such that $E_{n} v$ is the solution to (3.3) with $f=0$ and initial data $v \in L_{2}$. Let $\left\{\varphi_{i}\right\}$ and $\left\{\lambda_{i}\right\}$ be eigenfunctions and corresponding eigenvalues such that

$$
a\left(\varphi_{i}, v\right)=\lambda_{i}\left(\varphi_{i}, v\right)_{c}, \quad \forall v \in V
$$

It follows that the eigenvalues $\left\{\lambda_{i}\right\}$ are real and positive and $\left\{\varphi_{i}\right\}$ are orthogonal with respect to the inner products $(\cdot, \cdot)_{c}$ and $a(\cdot, \cdot)$. Furthermore, there is a finite subset of eigenfunctions that spans $V_{h}$, i.e., $\operatorname{span}\left\{\varphi_{i}\right\}_{i=1}^{M}=V_{h}$ for some $M<\infty$. With this notation, the solution $E_{n} v$ can be written as

$$
E_{n} v=\sum_{i=1}^{M} \frac{1}{\left(1+\tau \lambda_{i}\right)^{n}}\left(v, \varphi_{i}\right)_{c} \varphi_{i}
$$

The bounds now follows from [18, Lemma 7.3].

\subsection{Orthogonal decomposition}

In this section we describe the orthogonal decomposition which defines the GFEM space denoted $\hat{V}$ in the discussion above. We refer to $[13,14]$ for details. The GFEM space is defined using only the diffusion coefficient $A$, that is, the variations in $c$ are not accounted for in the construction of the space. In Sect. 4 we prove that this space indeed is sufficient to obtain convergence of the method.

For the construction of the GFEM space we use the (weighted) Clément interpolation operator introduced in [4], $\mathfrak{I}_{H}: V_{h} \rightarrow V_{H}$ defined by

$$
\mathfrak{I}_{H} v=\sum_{x \in \mathcal{N}}\left(\mathfrak{I}_{H} v\right)(x) \varphi_{x}, \quad \text { where }\left(\mathfrak{I}_{H} v\right)(x):=\frac{\int_{\Omega} v \varphi_{x}}{\int_{\Omega} \varphi_{x}} .
$$

For this interpolation operator the following result is proved [4]

$$
H_{K}^{-1}\left\|v-\mathfrak{I}_{H} v\right\|_{L_{2}(K)}+\left\|\nabla\left(v-\mathfrak{I}_{H} v\right)\right\|_{L_{2}(K)} \leq C\|\nabla v\|_{L_{2}\left(\bar{\omega}_{K}\right)}, \forall v \in V
$$

where $\bar{\omega}_{K}:=\cup\left\{\bar{K} \in \mathcal{T}_{H}: \bar{K} \cap K \neq \emptyset\right\}$ and $C$ depends on the shape regularity $\varrho$.

Let $V^{\mathrm{f}}=\left\{v \in V_{h}: \mathfrak{I}_{H} v=0\right\}$ be the kernel of the Clément interpolation operator (3.7). This space contains all fine scale features not resolved by $V_{H}$. The space $V_{h}$ can then be decomposed into $V_{h}=V_{H} \oplus V^{\mathrm{f}}$, where $v \in V_{h}$ can be written as a sum $v=v_{H}+v^{\mathrm{f}}$, with $v_{H} \in V_{H}, v^{\mathrm{f}} \in V^{\mathrm{f}}$, and $\left(v_{H}, v^{\mathrm{f}}\right)=0$.

Now define the orthogonal projection $R^{\mathrm{f}}: V_{h} \rightarrow V^{\mathrm{f}}$ by

$$
a\left(R^{\mathrm{f}} v, w\right)=a(v, w) \quad \forall w \in V^{\mathrm{f}}, v \in V_{h} .
$$

Using this projection we define the GFEM space, also referred to as the multiscale space, 


$$
V^{\mathrm{ms}}:=V_{H}-R^{\mathrm{f}} V_{H}
$$

which leads to another orthogonal decomposition $V_{h}=V^{\mathrm{ms}} \oplus V^{\mathrm{f}}$. Hence any function $v \in V_{h}$ has a unique decomposition $v=v^{\mathrm{ms}}+v^{\mathrm{f}}$, with $v^{\mathrm{ms}} \in V^{\mathrm{ms}}$ and $v^{\mathrm{f}} \in V^{\mathrm{f}}$, with $a\left(v^{\mathrm{ms}}, v^{\mathrm{f}}\right)=0$.

To define a basis for $V^{\mathrm{ms}}$ we want to find the projection $R^{\mathrm{f}}$ of the nodal basis function $\varphi_{x} \in V_{H}$. Let this projection be denoted $\phi_{x}$, so that $\phi_{x} \in V^{\mathrm{f}}$ satisfies the (global) corrector problem

$$
a\left(\phi_{x}, w\right)=a\left(\varphi_{x}, w\right), \quad \forall w \in V^{\mathrm{f}}
$$

A basis for the multiscale space $V^{\mathrm{ms}}$ is thus given by

$$
\left\{\varphi_{x}-\phi_{x}: x \in \mathcal{N}\right\}
$$

We also introduce the projection $R^{\mathrm{ms}}: V_{h} \rightarrow V^{\mathrm{ms}}$, defined by

$$
a\left(R^{\mathrm{ms}} v, w\right)=a(v, w), \quad \forall w \in V^{\mathrm{ms}}, v \in V_{h}
$$

Note that $R^{\mathrm{ms}}=I-R^{\mathrm{f}}$. For $R^{\mathrm{ms}}$ we have the following lemma, based on the results in [13].

Lemma 3.2 For the projection $R^{\mathrm{ms}}$ in (3.10) and $v \in V_{h}$ we have the error bound

$$
\left\|v-R^{\mathrm{ms}} v\right\| \leq C H^{2}\left\|\mathcal{A}_{h} v\right\|, \quad v \in V_{h},
$$

where $C$ depends on $\alpha_{1}$ and $\varrho$, but not on the variations of $A$ or $c$.

Proof Define the following elliptic auxiliary problem: find $z \in V_{h}$ such that

$$
a(z, w)=\left(v-R^{\mathrm{ms}} v, w\right), \quad \forall w \in V_{h} .
$$

In [13, Lemma 3.1] it was proven that the solution to an elliptic equation of the form

$$
a(u, w)=(g, w), \quad \forall w \in V_{h},
$$

satisfies the error estimate

$$
\|\| u-R^{\mathrm{ms}} u\|\mid \leq C H\| g \|,
$$

where $C$ depends on $\varrho$ and $\alpha_{1}$, but not on the variations of $A$. Hence, we have the following bound for $z$,

$$
\left\|z-R^{\mathrm{ms}} z\right\| \mid \leq C H\left\|v-R^{\mathrm{ms}} v\right\| .
$$


Furthermore, we note that $v-R^{\mathrm{ms}} v \in V_{h}$ and

$$
\begin{aligned}
\left\|v-R^{\mathrm{ms}} v\right\|^{2} & =\left(v-R^{\mathrm{ms}} v, v-R^{\mathrm{ms}} v\right)=a\left(z, v-R^{\mathrm{ms}} v\right) \\
& =a\left(z-R^{\mathrm{ms}} z, v-R^{\mathrm{ms}} v\right) \leq\left\|z-R^{\mathrm{ms}} z\right\|\|\|-R^{\mathrm{ms}} v\|\|
\end{aligned}
$$

Now, since $a(v, w)=\left(\mathcal{A}_{h} v, w\right)$, we get $\left\|v-R^{\mathrm{ms}} v\right\| \leq C H\left\|\mathcal{A}_{h} v\right\|$ and (3.11) follows.

In particular, if $U_{n}$ is the solution to (3.3), then (3.11) gives

$$
\begin{aligned}
\left\|U_{n}-R^{\mathrm{ms}} U_{n}\right\| & \leq C H^{2}\left\|P_{h}\left(f_{n}-c \bar{\partial}_{t} U_{n}\right)\right\|, \quad n \geq 1, \\
\left\|\bar{\partial}_{t} U_{n}-R^{\mathrm{ms}} \bar{\partial}_{t} U_{n}\right\| & \leq C H^{2}\left\|P_{h}\left(\bar{\partial}_{t} f_{n}-c \bar{\partial}_{t} \bar{\partial}_{t} U_{n}\right)\right\|, \quad n \geq 2,
\end{aligned}
$$

where $P_{h}$ is the $L_{2}$-projection onto $V_{h}$.

The result in Lemma 3.2 should be compared with the error of the classical Ritz projection $R_{h}: V \rightarrow V_{h}$ defined by $a\left(R_{h} v, w\right)=a(v, w), \forall w \in V_{h}$. Using elliptic regularity estimates, one achieves

$$
\left\|R_{h} v-v\right\| \leq C h^{2}\left\|D^{2} v\right\| \leq C h^{2}\|\mathcal{A} v\|,
$$

which is similar to the result in Lemma 3.2. However, in this case, $C$ depends on the variations of $A$ and the regularity of $\Omega$. This is avoided by using the $R^{\mathrm{ms}}$-projection, since the constant in Lemma 3.2 does not depend on the variations of $A$ or $c$.

Now define the corresponding GFEM to problem (3.3); find $U_{n}^{\mathrm{ms}} \in V^{\mathrm{ms}}$ such that

$$
\begin{aligned}
\left(\bar{\partial}_{t} U_{n}^{\mathrm{ms}}, v\right)_{c}+a\left(U_{n}^{\mathrm{ms}}, v\right) & =\left(f_{n}, v\right), \quad \forall v \in V^{\mathrm{ms}}, \\
\left(U_{0}^{\mathrm{ms}}, v\right)_{c} & =\left(U_{0}, v\right)_{c}, \quad \forall v \in V^{\mathrm{ms}},
\end{aligned}
$$

for $n=1, \ldots, N$. Furthermore, we define the operator $\mathcal{A}^{\mathrm{ms}}: V^{\mathrm{ms}} \rightarrow V^{\mathrm{ms}}$ by

$$
\left(\mathcal{A}^{\mathrm{ms}} v, w\right)=a(v, w), \quad \forall v, w \in V^{\mathrm{ms}} .
$$

Remark 3.3 In this remark we discuss the possibilities of including time dependency in the coefficients $c$ and $A$.

(i) It is possible, with a slight modification of the error analysis, to let $c=c(x, t)$ be time dependent with rapid variations in space. However, for simplicity, we shall only study the time independent case here.

(ii) We emphasize that the construction of the method in (3.12) depends on the fact that the diffusion coefficient does not depend on time. If we have $A=A(x, t)$, the multiscale space could be updated in each time step. For each $t_{n}$, we would then define a new Ritz projection $R^{\mathrm{f}}$ with $A=A\left(x, t_{n}\right)$ leading to a space $V_{n}^{\mathrm{ms}}$. If the variations in time are slow or periodic, it is also possible reuse the space for several time steps. However, if the variations are fast and non-periodic, then updating the basis may become too expensive which calls for a different approach. 


\subsection{Localization}

Since the corrector problems (3.9) are posed in the fine scale space $V^{\mathrm{f}}$ they are computationally expensive to solve. Moreover, the correctors $\phi_{x}$ generally have global support, which destroys the sparsity of the resulting linear system (3.12). However, as shown in [13], $\phi_{x}$ decays exponentially fast away from $x$. This observation motivates a localization of the corrector problems to smaller patches of coarse elements. Here we use a variant presented in [6], which reduces the required size of the patches.

We first define the notion of patches and their sizes. For all $K \in \mathcal{T}_{H}$ we define $\omega_{k}(K)$ to be the patch of size $k$, where

$$
\begin{aligned}
& \omega_{0}(K):=\operatorname{int} K, \\
& \omega_{k}(K):=\operatorname{int}\left(\cup\left\{\hat{K} \in \mathcal{T}_{H}: \hat{K} \cap \overline{\omega_{k-1}(K)} \neq \emptyset\right\}\right), \quad k=1,2, \ldots
\end{aligned}
$$

Moreover, we define $V^{\mathrm{f}}\left(\omega_{k}(K)\right):=\left\{w \in V^{\mathrm{f}}: v(z)=0\right.$ on $\left.\bar{\Omega} \backslash \omega_{k}(K)\right\}$.

Now define the operator $R_{K}^{\mathrm{f}}: V_{h} \rightarrow V^{\mathrm{f}}$ by

$$
\int_{\Omega} A \nabla R_{K}^{\mathrm{f}} v \cdot \nabla w=\int_{K} A \nabla v \cdot \nabla w, \quad \forall v \in V_{h}, w \in V^{\mathrm{f}},
$$

and note that $R^{\mathrm{f}}:=\sum_{K \in \mathcal{T}_{H}} R_{K}^{\mathrm{f}}$. We now localize the operator $R_{K}^{\mathrm{f}}$ by defining $R_{K, k}^{\mathrm{f}}: V_{h} \rightarrow V^{\mathrm{f}}\left(\omega_{k}(K)\right)$ through

$$
\int_{\omega_{k}(K)} A \nabla R_{K, k}^{\mathrm{f}} v \cdot \nabla w=\int_{K} A \nabla v \cdot \nabla w, \quad \forall v \in V_{h}, w \in V^{\mathrm{f}}\left(\omega_{k}(K)\right),
$$

and we define $R_{k}^{\mathrm{f}}:=\sum_{K \in \mathcal{T}_{H}} R_{K, k}^{\mathrm{f}}$. Hence we can, for each nonnegative integer $k$, define a localized multiscale space

$$
V_{k}^{\mathrm{ms}}:=V_{H}-R_{k}^{\mathrm{f}} V_{H}
$$

Here the basis is given by $\left\{\varphi_{x}-\phi_{k, x}: x \in \mathcal{N}\right\}$, where $\phi_{k, x}=R_{k}^{\mathrm{f}} \varphi_{x}$ is the localized version of $\phi_{x}$. The procedure of decomposing $V_{h}$ into the orthogonal spaces $V^{\mathrm{ms}}$ and $V^{\mathrm{f}}$ together with the localization of $V^{\mathrm{ms}}$ to $V_{k}^{\mathrm{ms}}$ is referred to as local orthogonal decomposition.

The following lemma follows from Lemma 3.6 in [6].

Lemma 3.4 There exists a constant $0<\mu<1$ that depends on the contrast $\alpha_{2} / \alpha_{1}$ such that

$$
\left|\left\|R^{\mathrm{f}} v-R_{k}^{\mathrm{f}} v\left|\left\|\leq C k^{d / 2} \mu^{k}|\|v\||, \quad \forall v \in V_{h},\right.\right.\right.\right.
$$

where $C$ depends on $\alpha_{1}, \alpha_{2}$, and $\varrho$, but not on the variations of $A$ or $c$. 
Now let $R_{k}^{\mathrm{ms}}: V_{h} \rightarrow V_{k}^{\mathrm{ms}}$ be the orthogonal projection defined by

$$
a\left(R_{k}^{\mathrm{ms}} v, w\right)=a(v, w), \quad \forall w \in V_{k}^{\mathrm{ms}} .
$$

The next lemma is a consequence of Theorem 3.7 in [6] and estimates the error due to the localization procedure.

Lemma 3.5 For the projection $R_{k}^{\mathrm{ms}}$ in (3.14) we have the bound

$$
\left\|v-R_{k}^{\mathrm{ms}} v\right\| \leq C\left(H+k^{d / 2} \mu^{k}\right)^{2}\left\|\mathcal{A}_{h} v\right\|, \quad \forall v \in V_{h} .
$$

Here $C$ depends on $\alpha_{1}, \alpha_{2}$, and $\varrho$, but not on the variations of $A$ or $c$.

Proof The proof is similar to the proof of Lemma 3.2. Let $z \in V_{h}$ be the solution to the elliptic dual problem

$$
a(z, w)=\left(v-R_{k}^{\mathrm{ms}} v, w\right), \quad \forall w \in V_{h},
$$

which gives

$$
\begin{aligned}
\left\|v-R_{k}^{\mathrm{ms}} v\right\|^{2} & =\left(v-R_{k}^{\mathrm{ms}} v, v-R_{k}^{\mathrm{ms}} v\right)=a\left(z-R_{k}^{\mathrm{ms}} z, v-R_{k}^{\mathrm{ms}} v\right) \\
& \leq\left\|z-R_{k}^{\mathrm{ms}} z\right\|\|\| v-R_{k}^{\mathrm{ms}} v\|\| .
\end{aligned}
$$

It follows from Theorem 3.7 in [6] that there exists a constant $C$ depending on $\alpha_{2}, \alpha_{1}$, and $\varrho$, such that $\left\|z-R_{k}^{\mathrm{ms}} z\right\| \mid \leq C\left(H+k^{d / 2} \mu^{k}\right)\left\|v-R_{k}^{\mathrm{ms}} v\right\|$, with $\mu$ as in Lemma 3.4. Since $\left(\mathcal{A}_{h} v, w\right)=a(v, w)$ we get $\left\|v-R_{k}^{\mathrm{ms}} v\right\| \leq C\left(H+k^{d / 2} \mu^{k}\right)\left\|\mathcal{A}_{h} v\right\|$ and (3.15) follows.

We are now ready to formulate the localized version of (3.12) by replacing $V^{\mathrm{ms}}$ by $V_{k}^{\mathrm{ms}}$. The localized GFEM formulation reads; find $U_{k, n}^{\mathrm{ms}} \in V_{k}^{\mathrm{ms}}$ such that

$$
\begin{aligned}
\left(\bar{\partial}_{t} U_{k, n}^{\mathrm{ms}}, v\right)_{c}+a\left(U_{k, n}^{\mathrm{ms}}, v\right) & =\left(f_{n}, v\right), \quad \forall v \in V_{k}^{\mathrm{ms}}, \\
\left(U_{k, 0}^{\mathrm{ms}}, v\right)_{c} & =\left(U_{0}, v\right)_{c}, \quad \forall v \in V_{k}^{\mathrm{ms}},
\end{aligned}
$$

for $n=1, \ldots, N$. We also define the operator $\mathcal{A}_{k}^{\mathrm{ms}}: V_{k}^{\mathrm{ms}} \rightarrow V_{k}^{\mathrm{ms}}$ by a localized version of (3.13)

$$
\left(\mathcal{A}_{k}^{\mathrm{ms}} v, w\right)=a(v, w), \quad \forall v, w \in V_{k}^{\mathrm{ms}} .
$$

We also define the solution operator $E_{k, n}^{\mathrm{ms}}$, such that the solution to (3.16), with $f=0$, can be expressed as $U_{k, n}^{\mathrm{ms}}=E_{k, n}^{\mathrm{ms}} U_{k, 0}^{\mathrm{ms}}$. For this operator we have estimates similar to (3.6). Since the initial data in (3.16) is the projection onto $V_{k}^{\mathrm{ms}}$ with respect to the inner product $(\cdot, \cdot)_{c}$, we define $P_{c, k}^{\mathrm{ms}}: L_{2} \rightarrow V_{k}^{\mathrm{ms}}$ by

$$
\left(P_{c, k}^{\mathrm{ms}} v, w\right)_{c}=(v, w)_{c}, \quad \forall w \in V_{k}^{\mathrm{ms}},
$$

to state the next lemma. 
Lemma 3.6 For $l=0,1$, and $v \in L_{2}$, we have

$$
\left\|\bar{\partial}_{t}^{l} E_{k, n}^{\mathrm{ms}} P_{c, k}^{\mathrm{ms}} v\right\| \leq C t_{n}^{-l}\|v\|, \quad n \geq l, \quad\left\|E_{k, n}^{\mathrm{ms}} P_{c, k}^{\mathrm{ms}} v\right\| \leq C t_{n}^{-1 / 2}\|v\|, \quad n \geq 1,
$$

where $C$ depends on the constant $\alpha_{1}, \gamma_{1}, \gamma_{2}$, but not on the variations of $A$ or $c$.

Proof As in the proof of Theorem 3.1 there exist a finite number of positive eigenvalues $\left\{\lambda_{i}\right\}_{i=1}^{M}$ and corresponding orthogonal eigenvectors $\left\{\varphi_{i}\right\}_{i=1}^{M}$ such that $\operatorname{span}\left\{\varphi_{i}\right\}=V_{k}^{\mathrm{ms}}$ and

$$
a\left(\varphi_{i}, v\right)=\lambda_{i}\left(\varphi_{i}, v\right)_{c}, \quad \forall v \in V
$$

It follows that $E_{k, n}^{\mathrm{ms}} v$ can be written as

$$
E_{k, n}^{\mathrm{ms}} v=\sum_{i=1}^{M} \frac{1}{\left(1+\tau \lambda_{i}\right)^{n}}\left(v, \varphi_{i}\right)_{c} \varphi_{i},
$$

and the bounds now follow from [18, Lemma 7.3].

\section{Error analysis}

In this section we derive error estimates for the local orthogonal decomposition method introduced in Sect. 3. The bounds of the time derivatives of a parabolic problem with nonsmooth initial data, (c.f. Theorem 3.1), depends on negative powers of $t_{n}$, which leads to error bounds containing negative powers of $t_{n}$. These are non-uniform in time, but of optimal order for a fix time $t_{n}>0$. The same phenomenon appears in classical finite element analysis for equations with nonsmooth initial data, see [18] and references therein. The error analysis in this section is carried out by only taking the $L_{2}$-norm of $U_{0}$, which allows $u_{0} \in L_{2}$.

Theorem 4.1 Let $U_{n}$ be the solution to (3.3) and $U_{k, n}^{\mathrm{ms}}$ the solution to (3.16). Then, for $1 \leq n \leq N$,

$$
\begin{aligned}
\left\|U_{k, n}^{\mathrm{ms}}-U_{n}\right\| \leq & C\left(1+\log \frac{t_{n}}{\tau}\right)\left(H+k^{d / 2} \mu^{k}\right)^{2}\left(t_{n}^{-1}\left\|U_{0}\right\|+\|f\|_{L_{\infty}\left(L_{2}\right)}\right. \\
& \left.+\|\dot{f}\|_{L_{\infty}\left(L_{2}\right)}\right)
\end{aligned}
$$

where $C$ depends on $\alpha_{1}, \alpha_{2}, \gamma_{1}, \gamma_{2}, \varrho$, and $T$, but not on the variations of $A$ or $c$.

The proof of Theorem 4.1 is divided into several lemmas. To study the error in the homogeneous case, $f=0$, we use techniques similar to the classical finite element analysis of problems with nonsmooth initial data, see [18] and the references therein.

Define $T_{h}: L_{2} \rightarrow V_{h}$ and $T_{k}^{\mathrm{ms}}: L_{2} \rightarrow V_{k}^{\mathrm{ms}}$ by

$$
\begin{gathered}
a\left(T_{h} v, w\right)=(v, w)_{c}, \quad \forall w \in V_{h}, v \in L_{2}, \\
a\left(T_{k}^{\mathrm{ms}} v, w\right)=(v, w)_{c}, \quad \forall w \in V_{k}^{\mathrm{ms}}, v \in L_{2} .
\end{gathered}
$$


With this notation the solution to the parabolic problem (3.3), with $f=0$, can be expressed as $T_{h} \bar{\partial}_{t} U_{n}+U_{n}=0$, since

$$
a\left(T_{h} \bar{\partial}_{t} U_{n}, w\right)=\left(\bar{\partial}_{t} U_{n}, w\right)_{c}=-a\left(U_{n}, w\right), \quad \forall w \in V_{h}
$$

Similarly, the solution to (3.16), with $f=0$, can be expressed as $T_{k}^{\mathrm{ms}} \bar{\partial}_{t} U_{k, n}^{\mathrm{ms}}+U_{k, n}^{\mathrm{ms}}=$ 0 . Note that $T_{h}$ and $T_{k}^{\mathrm{ms}}$ are self-adjoint and positive semi-definite with respect to $(\cdot, \cdot)_{c}$ on $L_{2}$, and $T_{k}^{\mathrm{ms}}=R_{k}^{\mathrm{ms}} T_{h}$.

Now, let $e_{n}=U_{k, n}^{\mathrm{ms}}-U_{n}$, where $e_{n}$ solves the error equation

$$
T_{k}^{\mathrm{ms}} \bar{\partial}_{t} e_{n}+e_{n}=-U_{n}-T_{k}^{\mathrm{ms}} \bar{\partial}_{t} U_{n}=\left(T_{h}-T_{k}^{\mathrm{ms}}\right) \bar{\partial}_{t} U_{n}=\left(R_{k}^{\mathrm{ms}}-I\right) U_{n}=: \rho_{n},
$$

for $n=1, \ldots, N$ with $T_{k}^{\mathrm{ms}} e_{0}=0$, since

$$
a\left(T_{k}^{\mathrm{ms}} e_{0}, w\right)=\left(U_{k}^{\mathrm{ms}}-U_{0}, w\right)_{c}=0
$$

The following lemma is a discrete version of [18, Lemma 3.3].

Lemma 4.2 Suppose $e_{n}$ satisfies the error equation (4.1). Then

$$
\begin{aligned}
\left\|e_{n}\right\|^{2} & \leq C\left(\left\|\rho_{n}\right\|^{2}+t_{n}^{-1}\left(\sum_{j=1}^{n} \tau\left\|\rho_{j}\right\|^{2}+\sum_{j=2}^{n} \tau t_{j}^{2}\left\|\bar{\partial}_{t} \rho_{j}\right\|^{2}\right)\right), \quad n \geq 2, \\
\left\|e_{1}\right\| & \leq C\left\|\rho_{1}\right\|
\end{aligned}
$$

where $C$ depends on $\gamma_{1}$ and $\gamma_{2}$, but not on the variations of $A$ or $c$.

Proof Multiply the error equation (4.1) by $c \bar{\partial}_{t} e_{n}$ and integrate over $\Omega$ to get

$$
\left(T_{k}^{\mathrm{ms}} \bar{\partial}_{t} e_{n}, \bar{\partial}_{t} e_{n}\right)_{c}+\left(e_{n}, \bar{\partial}_{t} e_{n}\right)_{c}=\left(\rho_{n}, \bar{\partial}_{t} e_{n}\right)_{c}
$$

where the first term on the left hand side is nonnegative, since $T_{k}^{\mathrm{ms}}$ is positive semidefinite on $L_{2}$. Multiplying by $\tau t_{n}$ we have

$$
t_{n}\left\|c^{1 / 2} e_{n}\right\|^{2}-t_{n}\left(e_{n}, e_{n-1}\right)_{c} \leq t_{n}\left(\rho_{n}, e_{n}-e_{n-1}\right)_{c}
$$

which gives

$$
\begin{aligned}
\frac{t_{n}}{2}\left\|c^{1 / 2} e_{n}\right\|^{2}-\frac{t_{n-1}}{2}\left\|c^{1 / 2} e_{n-1}\right\|^{2} \leq & t_{n}\left(\rho_{n}, e_{n}-e_{n-1}\right)_{c}+\frac{t_{n}-t_{n-1}}{2}\left\|c^{1 / 2} e_{n-1}\right\|^{2} \\
\leq & t_{n}\left(\rho_{n}, e_{n}\right)_{c}-t_{n-1}\left(\rho_{n-1}, e_{n-1}\right)_{c} \\
& -\left(t_{n} \rho_{n}-t_{n-1} \rho_{n-1}, e_{n-1}\right)_{c}+\frac{\tau}{2}\left\|c^{1 / 2} e_{n-1}\right\|^{2} .
\end{aligned}
$$


Summing over $n$ now gives

$$
\begin{aligned}
t_{n}\left\|c^{1 / 2} e_{n}\right\|^{2}-t_{1}\left\|c^{1 / 2} e_{1}\right\|^{2} \leq & 2 t_{n}\left(\rho_{n}, e_{n}\right)_{c}-2 t_{1}\left(\rho_{1}, e_{1}\right)_{c} \\
& -\sum_{j=2}^{n} 2\left(t_{j} \rho_{j}-t_{j-1} \rho_{j-1}, e_{j-1}\right)_{c}+\sum_{j=2}^{n} \tau\left\|c^{1 / 2} e_{j-1}\right\|^{2},
\end{aligned}
$$

and thus,

$$
\begin{aligned}
t_{n}\left\|c^{1 / 2} e_{n}\right\|^{2} \leq & C\left(t_{n}\left\|c^{1 / 2} \rho_{n}\right\|^{2}+\sum_{j=2}^{n} \tau\left(t_{j}^{2}\left\|c^{1 / 2} \bar{\partial}_{t} \rho_{j}\right\|^{2}+\left\|c^{1 / 2} \rho_{j-1}\right\|^{2}\right)\right. \\
& \left.+\sum_{j=2}^{n} \tau\left\|c^{1 / 2} e_{j-1}\right\|^{2}\right) .
\end{aligned}
$$

To estimate the last sum we note that, since $T_{k}^{\mathrm{ms}}$ is self-adjoint and positive semidefinite,

$$
\begin{aligned}
2\left(T_{k}^{\mathrm{ms}} \bar{\partial}_{t} e_{n}, e_{n}\right)_{c} & =\left(T_{k}^{\mathrm{ms}} \bar{\partial}_{t} e_{n}, e_{n}\right)_{c}+\left(T_{k}^{\mathrm{ms}} e_{n}, \bar{\partial}_{t} e_{n}\right)_{c} \\
& =\bar{\partial}_{t}\left(T_{k}^{\mathrm{ms}} e_{n}, e_{n}\right)_{c}+\tau\left(T_{k}^{\mathrm{ms}} \bar{\partial}_{t} e_{n}, \bar{\partial}_{t} e_{n}\right)_{c} \geq \bar{\partial}_{t}\left(T_{k}^{\mathrm{ms}} e_{n}, e_{n}\right)_{c}
\end{aligned}
$$

so by multiplying the error equation (4.1) by $2 c e_{n}$ we get

$$
\bar{\partial}_{t}\left(T_{k}^{\mathrm{ms}} e_{n}, e_{n}\right)_{c}+2\left\|c^{1 / 2} e_{n}\right\|^{2} \leq 2\left(T_{k}^{\mathrm{ms}} \bar{\partial}_{t} e_{n}, e_{n}\right)_{c}+2\left\|c^{1 / 2} e_{n}\right\|^{2}=2\left(\rho_{n}, e_{n}\right)_{c} .
$$

Multiplying by $\tau$ and summing over $n$ gives

$$
\left(T_{k}^{\mathrm{ms}} e_{n}, e_{n}\right)_{c}+\sum_{j=1}^{n} \tau\left\|c^{1 / 2} e_{j}\right\|^{2} \leq \sum_{j=1}^{n} \tau\left\|c^{1 / 2} \rho_{j}\right\|^{2}
$$

where we have used that $T_{k}^{\mathrm{ms}} e_{0}=0$. Since the first term is nonnegative we deduce that $\sum_{j=1}^{n} \tau\left\|c^{1 / 2} e_{j}\right\|^{2} \leq \sum_{j=1}^{n} \tau\left\|c^{1 / 2} \rho_{j}\right\|^{2}$ and (4.2) follows. For $n=1$ this also proves (4.3). Note that we have used the bounds on $c$ in (A2) to obtain the result in the $L_{2}$-norm.

Next lemma is a discrete version of a result that can be found in the proof of [18, Theorem 3.3].

Lemma 4.3 Under the assumptions of Lemma 4.2 we have, for $n \geq 2$, the bound

$$
\left\|e_{n}\right\| \leq C t_{n}^{-1}\left(\max _{2 \leq j \leq n} t_{j}^{2}\left\|\bar{\partial}_{t} \rho_{j}\right\|+\max _{1 \leq j \leq n}\left(t_{j}\left\|\rho_{j}\right\|+\left\|\sum_{r=1}^{j} \tau \rho_{r}\right\|\right)\right),
$$

where $C$ depends on $\gamma_{1}$ and $\gamma_{2}$, but not on the variations of $A$ or $c$. 
Proof It follows from Lemma 4.2 that

$$
\left\|e_{n}\right\| \leq C\left(\max _{2 \leq j \leq n} t_{j}\left\|\bar{\partial}_{t} \rho_{j}\right\|+\max _{1 \leq j \leq n}\left\|\rho_{j}\right\|\right), \quad n \geq 2,
$$

or by using Young's inequality with different constants the proof can be modified to show that

$$
\left\|e_{n}\right\| \leq \epsilon \max _{2 \leq j \leq n} t_{j}\left\|\bar{\partial}_{t} \rho_{j}\right\|+C(\epsilon) \max _{1 \leq j \leq n}\left\|\rho_{j}\right\|, \quad n \geq 2
$$

for some $\epsilon>0$. Now define $z_{j}=t_{j} e_{j}$. Then

$$
T_{k}^{\mathrm{ms}} \bar{\partial}_{t} z_{n}+z_{n}=t_{n} \rho_{n}+T_{k}^{\mathrm{ms}} e_{n-1}:=\eta_{n}, \quad n \geq 1,
$$

and, since $T_{k}^{\mathrm{ms}} z_{0}=0$ we conclude from Lemma 4.2

$$
\left\|z_{n}\right\| \leq \epsilon \max _{2 \leq j \leq n} t_{j}\left\|\bar{\partial}_{t} \eta_{j}\right\|+C \max _{1 \leq j \leq n}\left\|\eta_{j}\right\| .
$$

From the definition of $\eta_{j}$ it follows that

$$
\left\|\eta_{j}\right\| \leq t_{j}\left\|\rho_{j}\right\|+\left\|T_{k}^{\mathrm{ms}} e_{j-1}\right\|, \quad j \geq 1 \text {. }
$$

Furthermore, for $j \geq 2$

$$
\begin{aligned}
t_{j}\left\|\bar{\partial}_{t} \eta_{j}\right\| & \leq t_{j}\left\|\bar{\partial}_{t} t_{j} \rho_{j}\right\|+t_{j}\left\|\bar{\partial}_{t} T_{k}^{\mathrm{ms}} e_{j-1}\right\| \\
& \leq t_{j}^{2}\left\|\bar{\partial}_{t} \rho_{j}\right\|+t_{j}\left\|\rho_{j-1}\right\|+t_{j}\left\|\rho_{j-1}-e_{j-1}\right\| \\
& \leq t_{j}^{2}\left\|\bar{\partial}_{t} \rho_{j}\right\|+2 t_{j}\left\|\rho_{j}-\rho_{j-1}\right\|+2 t_{j}\left\|\rho_{j}\right\|+t_{j}\left\|e_{j-1}\right\| \\
& \leq 3 t_{j}^{2}\left\|\bar{\partial}_{t} \rho_{j}\right\|+2 t_{j}\left\|\rho_{j}\right\|+2 t_{j-1}\left\|e_{j-1}\right\| \\
& \leq C\left(t_{j}^{2}\left\|\bar{\partial}_{t} \rho_{j}\right\|+t_{j}\left\|\rho_{j}\right\|\right)+2\left\|z_{j-1}\right\|
\end{aligned}
$$

where we used $\frac{1}{2} t_{j} \leq t_{j-1} \leq t_{j}$ for $j \geq 2$. To bound $\left\|T_{k}^{\mathrm{ms}} e_{n}\right\|$ we define $\tilde{e}_{n}=$ $\sum_{j=1}^{n} \tau e_{j}$ and $\tilde{e}_{0}=0$. Multiplying the error equation (4.1) by $\tau$ and summing over $n$ gives

$$
\sum_{j=1}^{n} \tau T_{k}^{\mathrm{ms}} \bar{\partial}_{t} e_{j}+\tilde{e}_{n}=T_{k}^{\mathrm{ms}} \bar{\partial}_{t} \tilde{e}_{n}+\tilde{e}_{n}=\tilde{\rho}_{n}, \quad n \geq 1,
$$

where $\tilde{\rho}_{n}=\sum_{j=1}^{n} \tau \rho_{j}$ and we have used that $T_{k}^{\mathrm{ms}} e_{0}=0$. Note that by definition $T_{k}^{\mathrm{ms}} \tilde{e}_{0}=0$. Thus, by Lemma 4.2, we have

$$
\begin{aligned}
\left\|\tilde{e}_{n}\right\| & \leq C\left(\max _{2 \leq j \leq n} t_{j}\left\|\bar{\partial}_{t} \tilde{\rho}_{j}\right\|+\max _{1 \leq j \leq n}\left\|\tilde{\rho}_{j}\right\|\right) \\
& \leq C\left(\max _{2 \leq j \leq n} t_{j}\left\|\rho_{j}\right\|+\max _{1 \leq j \leq n}\left\|\sum_{r=1}^{j} \tau \rho_{r}\right\|\right) .
\end{aligned}
$$


Hence, since $T_{k}^{\mathrm{ms}} \bar{\partial}_{t} \tilde{e}_{n}=T_{k}^{\mathrm{ms}} e_{n}$,

$$
\left\|T_{k}^{\mathrm{ms}} e_{n}\right\| \leq\left\|\tilde{e}_{n}\right\|+\left\|\tilde{\rho}_{n}\right\| \leq C\left(\max _{2 \leq j \leq n} t_{j}\left\|\rho_{j}\right\|+\max _{1 \leq j \leq n}\left\|\sum_{r=1}^{j} \tau \rho_{r}\right\|\right) .
$$

With $\epsilon=\frac{1}{4}$ we get

$$
\begin{aligned}
\left\|z_{n}\right\| & \leq \frac{1}{4} \max _{2 \leq j \leq n} t_{j}\left\|\bar{\partial}_{t} \eta_{j}\right\|+C \max _{1 \leq j \leq n}\left\|\eta_{j}\right\| \\
& \leq \frac{1}{2} \max _{1 \leq j \leq n}\left\|z_{j}\right\|+C\left(\max _{2 \leq j \leq n} t_{j}^{2}\left\|\bar{\partial}_{t} \rho_{j}\right\|+\max _{1 \leq j \leq n}\left(t_{j}\left\|\rho_{j}\right\|+\left\|\sum_{r=1}^{j} \tau \rho_{r}\right\|\right)\right),
\end{aligned}
$$

but from (4.3) we deduce $\left\|z_{1}\right\| \leq t_{1}\left\|\rho_{1}\right\|$, and hence

$$
\left\|z_{n}\right\| \leq \frac{1}{2} \max _{2 \leq j \leq n}\left\|z_{j}\right\|+C\left(\max _{2 \leq j \leq n} t_{j}^{2}\left\|\bar{\partial}_{t} \rho_{j}\right\|+\max _{1 \leq j \leq n}\left(t_{j}\left\|\rho_{j}\right\|+\left\|\sum_{r=1}^{j} \tau \rho_{r}\right\|\right)\right)
$$

Choosing $n^{*}$ such that $\max _{2 \leq j \leq n} z_{j}=z_{n^{*}}$ we conclude (4.4).

Lemma 4.4 Assume $f=0$ and let $U_{k, n}^{\mathrm{ms}}$ be the solution to (3.16) and $U_{n}$ the solution to (3.3). Then, for $1 \leq n \leq N$,

$$
\left\|U_{k, n}^{\mathrm{ms}}-U_{n}\right\| \leq C\left(H+k^{d / 2} \mu^{k}\right)^{2} t_{n}^{-1}\left\|U_{0}\right\|
$$

where $C$ depends on $\alpha_{1}, \alpha_{2}, \gamma_{1}, \gamma_{2}, \varrho$, and $T$, but not on the variations of $A$ or $c$.

Proof From Lemma 4.3 we have

$$
\left\|e_{n}\right\| \leq C t_{n}^{-1}\left(\max _{2 \leq j \leq n} t_{j}^{2}\left\|\bar{\partial}_{t} \rho_{j}\right\|+\max _{1 \leq j \leq n}\left(t_{j}\left\|\rho_{j}\right\|+\left\|\sum_{r=1}^{j} \tau \rho_{r}\right\|\right)\right), \quad n \geq 2,
$$

and from Lemma $4.2\left\|e_{1}\right\| \leq C\left\|\rho_{1}\right\|$. The rest of the proof is based on estimates for the projection $R_{k}^{\mathrm{ms}}$ in Lemma 3.5 and the regularity of the homogeneous equation (3.6). We have

$$
\begin{aligned}
t_{j}^{2}\left\|\bar{\partial}_{t} \rho_{j}\right\| & \leq C\left(H+k^{d / 2} \mu^{k}\right)^{2} t_{j}^{2}\left\|\mathcal{A}_{h} \bar{\partial}_{t} U_{j}\right\| \\
& \leq C\left(H+k^{d / 2} \mu^{k}\right)^{2} t_{j}^{2}\left\|c^{1 / 2} \bar{\partial}_{t} \bar{\partial}_{t} U_{j}\right\| \leq C\left(H+k^{d / 2} \mu^{k}\right)^{2}\left\|U_{0}\right\|, \quad j \geq 2, \\
t_{j}\left\|\rho_{j}\right\| & \leq C\left(H+k^{d / 2} \mu^{k}\right)^{2} t_{j}\left\|\mathcal{A}_{h} U_{j}\right\| \\
& \leq C\left(H+k^{d / 2} \mu^{k}\right)^{2} t_{j}\left\|c^{1 / 2} \bar{\partial}_{t} U_{j}\right\| \leq C\left(H+k^{d / 2} \mu^{k}\right)^{2}\left\|U_{0}\right\|, \quad j \geq 1,
\end{aligned}
$$




$$
\begin{aligned}
\left\|\sum_{r=1}^{j} \tau \rho_{r}\right\| & =\left\|\sum_{r=1}^{j} \tau\left(T_{h}-T_{k}^{\mathrm{ms}}\right) \bar{\partial}_{t} U_{r}\right\| \leq\left\|\left(T_{h}-T_{k}^{\mathrm{ms}}\right)\left(U_{j}-U_{0}\right)\right\| \\
& \leq C\left(H+k^{d / 2} \mu^{k}\right)^{2}\left\|U_{0}\right\|,
\end{aligned}
$$

where we have used $T_{k}^{\mathrm{ms}}=R_{k}^{\mathrm{ms}} T_{h}$ and $\left\|U_{j}\right\| \leq C\left\|U_{0}\right\|$.

The next lemma concerns the convergence of the inhomogeneous parabolic problem (2.1) with initial data $U_{0}=0$.

Lemma 4.5 Assume $U_{0}=0$ and let $U_{k, n}^{\mathrm{ms}}$ be the solution to (3.16) and $U_{n}$ the solution to (3.3). Then, for $1 \leq n \leq N$,

$$
\left\|U_{k, n}^{\mathrm{ms}}-U_{n}\right\| \leq C\left(1+\log \frac{t_{n}}{\tau}\right)\left(H+k^{d / 2} \mu^{k}\right)^{2}\left(\|f\|_{L_{\infty}\left(L_{2}\right)}+\|\dot{f}\|_{L_{\infty}\left(L_{2}\right)}\right),
$$

where $C$ depends on $\alpha_{1}, \alpha_{2}, \gamma_{1}, \gamma_{2}, \varrho$, and $T$, but not on the variations of $A$ or $c$.

Proof Let $U_{k, n}^{\mathrm{ms}}-U_{n}=U_{k, n}^{\mathrm{ms}}-R_{k}^{\mathrm{ms}} U_{n}+R_{k}^{\mathrm{ms}} U_{n}-U_{n}=: \theta_{n}+\rho_{n}$. For $\rho_{n}$ we use Lemma 3.5 to achieve the estimate

$$
\left\|\rho_{n}\right\| \leq C\left(H+k^{d / 2} \mu^{k}\right)^{2}\left\|\mathcal{A}_{h} U_{n}\right\|
$$

Now, for $v \in V_{k}^{\mathrm{ms}}$ we have

$$
\left(\bar{\partial}_{t} \theta_{n}, v\right)_{c}+a\left(\theta_{n}, v\right)=\left(-\bar{\partial}_{t} \rho_{n}, v\right)_{c}
$$

Using Duhamel's principle we have

$$
\theta_{n}=\tau \sum_{j=1}^{n} E_{k, n-j+1}^{\mathrm{ms}} P_{c, k}^{\mathrm{ms}}\left(-\bar{\partial}_{t} \rho_{j}\right)
$$

since $\theta_{0}=0$. Summation by parts now gives

$$
\theta_{n}=E_{k, n}^{\mathrm{ms}} P_{c, k}^{\mathrm{ms}} \rho_{0}-P_{c, k}^{\mathrm{ms}} \rho_{n}-\tau \sum_{j=1}^{n} \bar{\partial}_{t} E_{k, n-j+1}^{\mathrm{ms}} P_{c, k}^{\mathrm{ms}} \rho_{j}
$$

Note that $\rho_{0}=0$. Using Lemma 3.5 and Lemma 3.6 we get

$$
\begin{aligned}
\left\|\theta_{n}\right\| & \leq C\left(\left\|\rho_{n}\right\|+\tau \sum_{j=1}^{n} t_{n-j+1}^{-1}\left\|\rho_{j}\right\|\right) \\
& \leq C\left(H+k^{d / 2} \mu^{k}\right)^{2} \max _{1 \leq j \leq n}\left\|\mathcal{A}_{h} U_{j}\right\|\left(1+\tau \sum_{j=1}^{n} t_{n-j+1}^{-1}\right),
\end{aligned}
$$


where the last sum can be bounded by

$$
\tau \sum_{j=1}^{n} t_{n-j+1}^{-1} \leq 1+\log \frac{t_{n}}{\tau} .
$$

It remains to bound $\left\|\mathcal{A}_{h} U_{n}\right\|$. We have $\mathcal{A}_{h} U_{n}=P_{h}\left(f_{n}-c \bar{\partial}_{t} U_{n}\right)$ and Theorem 3.1 gives

$$
\left\|\mathcal{A}_{h} U_{j}\right\| \leq C\left(\left\|f_{j}\right\|+\left\|\bar{\partial}_{t} U_{j}\right\|\right) \leq C\left(\|f\|_{L_{\infty}\left(L_{2}\right)}+\|\dot{f}\|_{L_{\infty}\left(L_{2}\right)}\right)
$$

which completes the proof.

Proof (of Theorem 4.1) The result follows from Lemmas 4.4 and 4.5 by rewriting $U_{n}=U_{n, 1}+U_{n, 2}$, where $U_{n, 1}$ is the solution to the homogeneous problem and $U_{n, 2}$ the solution to the inhomogeneous problem with vanishing initial data.

Remark 4.6 We note that the choice of $k$ and the size of $\mu$ determine the rate of the convergence. In general, to achieve optimal order convergence rate, $k$ should be chosen proportional to $\log \left(H^{-1}\right)$, i.e. $k=c \log \left(H^{-1}\right)$. With this choice of $k$ we have $\left\|U_{k, n}^{\mathrm{ms}}-U_{n}\right\| \leq C(1+\log n) H^{2} t_{n}^{-1}$.

\section{The semilinear parabolic equation}

In this section we discuss how the above techniques can be extended to a semilinear parabolic problem with multiscale diffusion coefficient. In this section we assume, for simplicity, that the coefficient $c=1$.

\subsection{Problem formulation}

We are interested in equations of the form

$$
\begin{aligned}
\dot{u}-\nabla \cdot(A \nabla u)=f(u), & \text { in } \Omega \times(0, T], \\
u=0, & \text { on } \partial \Omega \times(0, T], \\
u(\cdot, 0)=u_{0}, & \text { in } \Omega,
\end{aligned}
$$

where $f: \mathbb{R} \rightarrow \mathbb{R}$ is twice continuously differentiable and $\Omega$ is a polygonal/polyhedral boundary in $\mathbb{R}^{d}$, for $d \leq 3$. For $d=2,3, f$ is assumed to fulfill the growth condition

$$
\left|f^{(l)}(\xi)\right| \leq C\left(1+|\xi|^{\delta+1-l}\right), \quad \text { for } l=1,2,
$$

where $\delta=2$ if $d=3$ and $\delta \in[1, \infty)$ if $d=2$. Furthermore, we assume that the diffusion $A$ fulfills assumption (A1) and $u_{0} \in V$.

Example 5.1 The Allen-Cahn equation $\dot{u}-\nabla \cdot(A \nabla u)=-\left(u^{3}-u\right)$ fulfills the assumption (5.2). 
Define the ball $B_{R}:=\left\{v \in V:\|v\|_{H^{1}} \leq R\right\}$. Using Hölder and Sobolev inequalities the following lemma can be proved, see [12].

Lemma 5.2 If $f$ fulfills assumption (5.2) and $u, v \in B_{R}$, then

$\|f(u)\| \leq C,\left\|f^{\prime}(u) z\right\|_{H^{-1}} \leq C\|z\|,\left\|f^{\prime}(u) z\right\| \leq C\|z\|_{H^{1}},\left\|f^{\prime \prime}(u) z\right\|_{H^{-1}} \leq C\|z\|$,

and

$$
\|f(u)-f(v)\|_{H^{-1}} \leq C\|u-v\|,
$$

where $C$ is a constant depending on $R$.

From (5.1) we derive the variational form; find $u(t) \in V$ such that

$$
(\dot{u}, v)+(A \nabla u, \nabla v)=(f(u), v), \quad \forall v \in V,
$$

and $u(0)=u_{0}$. For this problem local existence of a solution can be derived given that the initial data $u_{0} \in V$, see [12].

Theorem 5.3 Assume that (A1) and (5.2) are satisfied. Then, for $u_{0} \in B_{R}$, there exist $T_{0}=T_{0}(R)$ and $C_{1}>0$, such that (5.3) has a unique solution $u \in C\left(0, T_{0} ; V\right)$ and $\|u\|_{L_{\infty}\left(0, T_{0} ; V\right)} \leq C_{1} R$.

For the Allen-Cahn equation it is possible to find an a priori global bound of $u$. This means that for any time $T$ there exists $R$ such that if $u$ is a solution then $\|u(t)\|_{L_{\infty}\left(H^{1}\right)} \leq R$ for $t \in[0, T]$. Thus we can apply the local existence theorem repeatedly to attain global existence, see [12].

\subsection{Numerical approximation}

The assumptions and definitions of the families of triangulations $\left\{\mathcal{T}_{h}\right\}_{h>0}$ and $\left\{\mathcal{T}_{H}\right\}_{H>h}$ and the corresponding spaces $V_{H}$ and $V_{h}$ remain the same as in Sect. 3. For the discretization in time we use a uniform time discretization given by

$$
0=t_{0}<t_{1}<\cdots<t_{N}=T_{0}, \quad \text { where } t_{n}-t_{n-1}=\tau,
$$

and $T_{0}$ is given from Theorem 5.3. With these discrete spaces we consider the semiimplicit backward Euler scheme where $U_{n} \in V_{h}$ satisfies

$$
\left(\bar{\partial}_{t} U_{n}, v\right)+\left(A \nabla U_{n}, \nabla v\right)=\left(f\left(U_{n-1}\right), v\right), \quad \forall v \in V_{h},
$$

for $n=1, \ldots, N$ where $U_{0} \in V_{h}$ is an approximation of $u_{0}$. It is proven in [11] that this scheme satisfies the bound

$$
\left\|U_{n}-u\left(t_{n}\right)\right\| \leq C t_{n}^{-1 / 2}\left(h^{2}+\tau\right),
$$


if we choose, for instance, $U_{0}=P_{h} u_{0}$, where $P_{h}$ denotes the $L_{2}$-projection onto $V_{h}$. Note that $C$ in this bound depends on the variations of $A$.

The following theorem gives some regularity estimates of the solution to (5.5).

Theorem 5.4 Assume that (A1) and (5.2) are satisfied. Then, for $U_{0} \in B_{R}$, there exist $T_{0}=T_{0}(R)$ and $C_{1}>0$ such that (5.5) has a unique solution $U_{n} \in V_{h}$, for $1 \leq n \leq N$, and $\max _{1 \leq n \leq N}\left\|U_{n}\right\|_{H^{1}} \leq C_{1} R$. Moreover, the following bounds hold

$\left\|\bar{\partial}_{t} U_{n}\right\| \leq C t_{n}^{-1 / 2}, n \geq 1, \quad\left\|\bar{\partial}_{t} U_{n} \mid\right\| \leq C t_{n}^{-1}, n \geq 1, \quad\left\|\bar{\partial}_{t} \bar{\partial}_{t} U_{n}\right\| \leq C t_{n}^{-3 / 2}, n \geq 2$,

where $C$ depends on $\alpha_{1}, T_{0}$, and $R$, but not on the variations of $A$.

Proof We only prove the estimate $\left\|\bar{\partial}_{t} \bar{\partial}_{t} U_{n}\right\| \leq C t_{n}^{-3 / 2}$ here. The other two follow by similar arguments.

From (5.5) we get

$$
\begin{aligned}
\left(\bar{\partial}_{t} \bar{\partial}_{t} U_{n}, v\right)+a\left(\bar{\partial}_{t} U_{n}, v\right) & =\left(\bar{\partial}_{t} f\left(U_{n-1}\right), v\right), \quad \forall v \in V_{h}, n \geq 2, \\
\left(\bar{\partial}_{t}^{(3)} U_{n}, v\right)+a\left(\bar{\partial}_{t} \bar{\partial}_{t} U_{n}, v\right) & =\left(\bar{\partial}_{t} \bar{\partial}_{t} f\left(U_{n-1}\right), v\right), \quad \forall v \in V_{h}, n \geq 3 .
\end{aligned}
$$

Choosing $v=\bar{\partial}_{t} \bar{\partial}_{t} U_{n}$ in (5.7) gives

$$
\frac{1}{\tau}\left\|\bar{\partial}_{t} \bar{\partial}_{t} U_{n}\right\|^{2}-\frac{1}{\tau}\left(\bar{\partial}_{t} \bar{\partial}_{t} U_{n-1}, \bar{\partial}_{t} \bar{\partial}_{t} U_{n}\right)+\left\|\bar{\partial}_{t} \bar{\partial}_{t} U_{n}\right\| \|^{2}=\left(\bar{\partial}_{t} \bar{\partial}_{t} f\left(U_{n-1}\right), \bar{\partial}_{t} \bar{\partial}_{t} U_{n}\right),
$$

which gives the bound

$$
\left\|\bar{\partial}_{t} \bar{\partial}_{t} U_{n}\right\|^{2}-\left\|\bar{\partial}_{t} \bar{\partial}_{t} U_{n-1}\right\|^{2} \leq C \tau\left\|\bar{\partial}_{t} \bar{\partial}_{t} f\left(U_{n-1}\right)\right\|_{H^{-1}}
$$

Using Lemma 5.2 we have for $\xi_{j} \in\left(\min \left\{U_{n-j}, U_{n-(j-1)}\right\}, \max \left\{U_{n-j}, U_{n-(j-1)}\right\}\right)$

$$
\begin{aligned}
\left\|\bar{\partial}_{t} \bar{\partial}_{t} f\left(U_{n}\right)\right\|_{H^{-1}}= & \frac{1}{\tau^{2}}\left\|f^{\prime}\left(\xi_{1}\right)\left(U_{n}-U_{n-1}\right)-f^{\prime}\left(\xi_{2}\right)\left(U_{n-1}-U_{n-2}\right)\right\|_{H^{-1}} \\
\leq & \frac{1}{\tau^{2}}\left\|\left(f^{\prime}\left(\xi_{1}\right)-f^{\prime}\left(\xi_{2}\right)\right)\left(U_{n}-U_{n-1}\right)\right\|_{H^{-1}} \\
& +\frac{1}{\tau^{2}}\left\|f^{\prime}\left(\xi_{2}\right)\left(U_{n}-2 U_{n-1}+U_{n-2}\right)\right\|_{H^{-1}} \\
\leq & \frac{1}{\tau^{2}}\left\|\left(\xi_{1}-\xi_{2}\right)\left(U_{n}-U_{n-1}\right)\right\|+C\left\|\bar{\partial}_{t} \bar{\partial}_{t} U_{n}\right\|
\end{aligned}
$$

Note that $\left|\xi_{1}-\xi_{2}\right| \leq\left|U_{n-2}-U_{n-1}\right|+\left|U_{n-1}-U_{n}\right|$. By using Sobolev embeddings we get

$$
\begin{aligned}
\frac{1}{\tau^{2}}\left\|\left(\xi_{1}-\xi_{2}\right)\left(U_{n}-U_{n-1}\right)\right\| & \leq \max _{n-1 \leq j \leq n} 2\left\|\left(\bar{\partial}_{t} U_{j}\right)^{2}\right\| \leq \max _{n-1 \leq j \leq n} 2\left\|\bar{\partial}_{t} U_{j}\right\|_{L_{4}}^{2} \\
& \leq C \max _{n-1 \leq j \leq n}\left\|\bar{\partial}_{t} U_{j}\right\|_{H^{1}}^{2} \leq C t_{n-1}^{-2} \leq C t_{n}^{-2}
\end{aligned}
$$


where we recall the bounds $\frac{1}{2} t_{j} \leq t_{j-1} \leq t_{j}$ for $j \geq 2$. Multiplying by $\tau t_{n}^{4}$ in (5.8) and summing over $n$ gives

$$
\begin{aligned}
t_{n}^{4}\left\|\bar{\partial}_{t} \bar{\partial}_{t} U_{n}\right\|^{2} \leq & C \sum_{j=3}^{n}\left(\tau t_{j}^{4}\left\|\bar{\partial}_{t} \bar{\partial}_{t} f\left(U_{j-1}\right)\right\|_{H^{-1}}^{2}+\left(t_{j}^{4}-t_{j-1}^{4}\right)\left\|\bar{\partial}_{t} \bar{\partial}_{t} U_{j-1}\right\|^{2}\right) \\
& +t_{2}^{4}\left\|\bar{\partial}_{t} \bar{\partial}_{t} U_{2}\right\|^{2} \\
\leq & C \sum_{j=3}^{n} \tau\left(t_{j}^{4}\left\|\bar{\partial}_{t} \bar{\partial}_{t} U_{j-1}\right\|^{2}+t_{j}^{4} t_{j-1}^{-4}+t_{j-1}^{3}\left\|\bar{\partial}_{t} \bar{\partial}_{t} U_{j-1}\right\|^{2}\right) \\
& +t_{2}^{4}\left\|\bar{\partial}_{t} \bar{\partial}_{t} U_{2}\right\|^{2} \\
\leq & C \sum_{j=3}^{n} \tau\left(t_{j-1}^{4}\left\|\bar{\partial}_{t} \bar{\partial}_{t} U_{j-1}\right\|^{2}+t_{j-1}^{3}\left\|\bar{\partial}_{t} \bar{\partial}_{t} U_{j-1}\right\|^{2}\right) \\
& +t_{2}^{4}\left\|\bar{\partial}_{t} \bar{\partial}_{t} U_{2}\right\|^{2}+C t_{n}
\end{aligned}
$$

for $n \geq 3$. Using $\left\|\bar{\partial}_{t} U_{j}\right\| \leq C t_{j}^{-1 / 2}$ for $j \geq 1$ we get

$$
t_{2}^{4}\left\|\bar{\partial}_{t} \bar{\partial}_{t} U_{2}\right\|^{2} \leq C \tau^{2}\left(\left\|\bar{\partial}_{t} U_{2}\right\|^{2}+\left\|\bar{\partial}_{t} U_{1}\right\|^{2}\right) \leq C \tau^{2}\left(t_{2}^{-1}+t_{1}^{-1}\right) \leq C \tau .
$$

Now, to bound $\sum_{j=2}^{n} t_{j}^{3}\left\|\bar{\partial}_{t} \bar{\partial}_{t} U_{j}\right\|$, we choose $v=\bar{\partial}_{t} \bar{\partial}_{t} U_{n}$ in (5.6) to derive

$$
\left\|\bar{\partial}_{t} \bar{\partial}_{t} U_{n}\right\|^{2}+\frac{1}{\tau}\left\|\bar{\partial}_{t} U_{n}\right\|^{2}-\frac{1}{\tau}\left\|\bar{\partial}_{t} U_{n-1}\right\|^{2} \leq\left\|\bar{\partial}_{t} f\left(U_{n-1}\right)\right\|^{2} .
$$

and with $\xi_{j}$ as above, we get

$$
\left\|\bar{\partial}_{t} f\left(U_{n-1}\right)\right\|=\left\|f^{\prime}\left(\xi_{2}\right) \bar{\partial}_{t} U_{n-1}\right\| \leq C\left\|\bar{\partial}_{t} U_{n-1}\right\| \mid \leq C t_{n-1}^{-1},
$$

where we used Lemma 5.2 and $\left\|\bar{\partial}_{t} U_{j}\right\| \mid \leq C t_{j}^{-1}$ for $j \geq 1$. Multiplying (5.9) with $\tau t_{n}^{3}$ and summing over $n$ gives

$$
\begin{aligned}
\sum_{j=2}^{n} \tau t_{j}^{3}\left\|\bar{\partial}_{t} \bar{\partial}_{t} U_{j}\right\|^{2}+t_{n}^{3}\left\|\bar{\partial}_{t} U_{n}\right\| \|^{2} \leq & C \sum_{j=2}^{n}\left(\tau t_{j}^{3} t_{j-1}^{-2}+\left.\left(t_{j}^{3}-t_{j-1}^{3}\right)\left\|\bar{\partial}_{t} U_{j-1}\right\|\right|^{2}\right) \\
& +t_{1}^{3}\|\| \bar{\partial}_{t} U_{1}\|\|^{2} \\
\leq & C \sum_{j=2}^{n}\left(\tau t_{j}+\tau t_{j-1}^{2}\left\|\bar{\partial}_{t} U_{j-1}\right\| \|^{2}\right)+t_{1}^{3}\left\|\bar{\partial}_{t} U_{1}\right\| \|^{2}
\end{aligned}
$$

Using $\left\|\bar{\partial}_{t} U_{j}\right\| \mid \leq C t_{j}^{-1}$ for $j \geq 1$ we get

$$
\sum_{j=2}^{n} \tau t_{j}^{3}\left\|\bar{\partial}_{t} \bar{\partial}_{t} U_{j}\right\|^{2} \leq C\left(t_{n}^{2}+t_{n}+t_{1}\right) \leq C t_{n}
$$


where $C$ now depends on $t_{n} \leq T$. So we have proved

$$
\begin{aligned}
t_{n}^{4}\left\|\bar{\partial}_{t} \bar{\partial}_{t} U_{n}\right\|^{2} & \leq C \sum_{j=3}^{n} \tau t_{j-1}^{4}\left\|\bar{\partial}_{t} \bar{\partial}_{t} U_{j-1}\right\|^{2}+C t_{n}+\tau \\
& \leq C \sum_{j=2}^{n-1} \tau t_{j}^{4}\left\|\bar{\partial}_{t} \bar{\partial}_{t} U_{j}\right\|^{2}+C t_{n+1} \leq C \sum_{j=2}^{n-1} \tau t_{j}^{4}\left\|\bar{\partial}_{t} \bar{\partial}_{t} U_{j}\right\|^{2}+C t_{n} .
\end{aligned}
$$

Applying the classical discrete Grönwall's lemma gives

$$
t_{n}^{4}\left\|\bar{\partial}_{t} \bar{\partial}_{t} U_{n}\right\|^{2} \leq C t_{n}
$$

which proves $\left\|\bar{\partial}_{t} \bar{\partial}_{t} U_{n}\right\| \leq C t_{n}^{-3 / 2}$ for $n \geq 3$. For $n=2$ we proved

$$
t_{2}^{4}\left\|\bar{\partial}_{t} \bar{\partial}_{t} U_{2}\right\|^{2} \leq C \tau \leq C t_{2}
$$

which completes the proof.

We use the same GFEM space as in Sect. 3, that is, $V^{\mathrm{ms}}=V_{H}-R^{\mathrm{f}} V_{H}$ and the localized version $V_{k}^{\mathrm{ms}}=V_{H}-R_{k}^{\mathrm{f}} V_{H}$. Furthermore, for the completely discrete scheme, we consider the time discretization defined in (5.4) and the linearized backward Euler method thus reads; find $U_{k, n}^{\mathrm{ms}} \in V^{\mathrm{ms}}$ such that $U_{k, 0}^{\mathrm{ms}}=P_{k}^{\mathrm{ms}} U_{0}$ and

$$
\left(\bar{\partial}_{t} U_{k, n}^{\mathrm{ms}}, v\right)+a\left(U_{k, n}^{\mathrm{ms}}, v\right)=\left(f\left(U_{k, n-1}^{\mathrm{ms}}\right), v\right)
$$

for $n=1, \ldots, N$ where $P_{k}^{\mathrm{ms}}$ is the $L_{2}$-projection onto $V_{k}^{\mathrm{ms}}$.

To derive an error estimates we represent the solution to (5.10) by using Duhamel's principle. Note that $U_{k, n}^{\mathrm{ms}}$ is the solution to the equation

$$
\bar{\partial}_{t} U_{k, n}^{\mathrm{ms}}+\mathcal{A}_{k}^{\mathrm{ms}} U_{k, n}^{\mathrm{ms}}=P_{k}^{\mathrm{ms}} f\left(U_{k, n-1}^{\mathrm{ms}}\right)
$$

and by Duhamel's principle we get

$$
U_{k, n}^{\mathrm{ms}}=E_{k, n}^{\mathrm{ms}} U_{k, 0}^{\mathrm{ms}}+\tau \sum_{j=1}^{n} E_{k, n-j+1}^{\mathrm{ms}} P_{k}^{\mathrm{ms}} f\left(U_{k, j-1}^{\mathrm{ms}}\right)
$$

Note that we use $c=1$ in the definition of the solution operator $E_{k, n}^{\mathrm{ms}}$ in Sect. 3.2.

\subsection{Error analysis}

For the error analysis we need the following generalized discrete Grönwall lemma, see, e.g., [12]. 
Lemma 5.5 Let $A, B \geq 0, \beta_{1}, \beta_{2}>0,0 \leq t_{0}<t_{n} \leq T$, and $0 \leq \varphi_{n} \leq R$. If

$$
\varphi_{n} \leq A t_{n}^{-1+\beta_{1}}+B \tau \sum_{j=1}^{n-1} t_{n-j+1}^{-1+\beta_{2}} \varphi_{j}
$$

then there is a constant $C$ depending on $B, \beta_{1}, \beta_{2}$, and, $T$, such that,

$$
\varphi_{n} \leq C A t_{n}^{-1+\beta_{1}} \text {. }
$$

Next lemma states a result for $\mathcal{A}_{k}^{\mathrm{ms}}$ which is needed in the analysis. A proof of the bound can be found in [12].

Lemma 5.6 The following bound holds

$$
\left\|\left(\mathcal{A}_{k}^{\mathrm{ms}}\right)^{-1 / 2} P_{k}^{\mathrm{ms}} f\right\| \leq C\|f\|_{H^{-1}}, \quad f \in L_{2},
$$

where $C$ depends on $\alpha_{1}$, but not on the variations of $A$.

Theorem 5.7 For given $R \geq 0$ and $T_{0}>0$ let $U_{n}$ be the solution to (5.5) and $U_{k, n}^{\mathrm{ms}}$ be the solution to (5.10), such that $U_{n}, U_{k, n}^{\mathrm{ms}} \in B_{R}$. Then, for $1 \leq n \leq N$,

$$
\left\|U_{k, n}^{\mathrm{ms}}-U_{n}\right\| \leq C\left(H+k^{d / 2} \mu^{k}\right)^{2} t_{n}^{-1 / 2},
$$

where $C$ depends on $\alpha_{1}, \alpha_{2}, \varrho, R$, and $T_{0}$, but not on the variations of $A$.

Proof First we define $e_{n}=U_{k, n}^{\mathrm{ms}}-U_{n}=\left(U_{k, n}^{\mathrm{ms}}-R_{k}^{\mathrm{ms}} U_{n}\right)+\left(R_{k}^{\mathrm{ms}} U_{n}-U_{n}\right)=\theta_{n}+\rho_{n}$. For $\rho_{j}$ we use Lemma 3.5 to prove the bounds

$$
\left\|\rho_{j}\right\| \leq C\left(H+k^{d / 2} \mu^{k}\right)^{2} t_{j}^{-1 / 2}, \quad j \geq 1,
$$

and

$$
\left\|\bar{\partial}_{t} \rho_{j}\right\| \leq C\left(H+k^{d / 2} \mu^{k}\right)^{2} t_{j}^{-3 / 2}, \quad j \geq 2 .
$$

For $\theta_{n}$ we have

$$
\theta_{n}=E_{k, n}^{\mathrm{ms}} \theta_{0}+\tau \sum_{j=1}^{n} E_{k, n-j+1}^{\mathrm{ms}} P_{k}^{\mathrm{ms}}\left(f\left(U_{k, j-1}^{\mathrm{ms}}\right)-f\left(U_{j-1}\right)-\bar{\partial}_{t} \rho_{j}\right) .
$$

To bound $\left\|\theta_{k, n}\right\|$ we first assume $n \geq 2$ and use summation by parts for the first part of the sum. Defining $n_{2}$ to be the integer part of $n / 2$ we can write

$$
\begin{gathered}
-\tau \sum_{j=1}^{n_{2}} E_{k, n-j+1}^{\mathrm{ms}} P_{k}^{\mathrm{ms}} \bar{\partial}_{t} \rho_{j}= \\
E_{k, n}^{\mathrm{ms}} P_{k}^{\mathrm{ms}} \rho_{0}-E_{k, n-n_{2}}^{\mathrm{ms}} P_{k}^{\mathrm{ms}} \rho_{n_{2}} \\
-\tau \sum_{j=1}^{n_{2}} \bar{\partial}_{t} E_{k, n-j+1}^{\mathrm{ms}} P_{k}^{\mathrm{ms}} \rho_{j},
\end{gathered}
$$


and $\theta_{n}$ can be rewritten as

$$
\begin{aligned}
\theta_{n}= & E_{k, n}^{\mathrm{ms}} P_{k}^{\mathrm{ms}} e_{0}-E_{k, n-n_{2}}^{\mathrm{ms}} P_{k}^{\mathrm{ms}} \rho_{n_{2}}-\tau \sum_{j=1}^{n_{2}} \bar{\partial}_{t} E_{k, n-j+1}^{\mathrm{ms}} P_{k}^{\mathrm{ms}} \rho_{j} \\
& -\tau \sum_{j=n_{2}+1}^{n} E_{k, n-j+1}^{\mathrm{ms}} P_{k}^{\mathrm{ms}} \bar{\partial}_{t} \rho_{j} \\
& +\tau \sum_{j=1}^{n}\left(\mathcal{A}_{k}^{\mathrm{ms}}\right)^{1 / 2} E_{k, n-j+1}^{\mathrm{ms}}\left(\mathcal{A}_{k}^{\mathrm{ms}}\right)^{-1 / 2} P_{k}^{\mathrm{ms}}\left(f\left(U_{k, j-1}^{\mathrm{ms}}\right)-f\left(U_{j-1}\right)\right)
\end{aligned}
$$

where we note that $P_{k}^{\mathrm{ms}} e_{0}=0$. To estimate these terms we need the following bounds for $\beta_{1}, \beta_{2}>0$

$$
\tau \sum_{j=1}^{n} t_{n-j+1}^{-1+\beta_{1}} t_{j}^{-1+\beta_{2}} \leq C_{\beta_{1}, \beta_{2}} t_{n}^{-1+\beta_{1}+\beta_{2}}, \quad \tau \sum_{j=1}^{n_{2}} t_{n-j+1}^{-\beta_{1}} t_{j}^{-1+\beta_{2}} \leq C_{\beta_{1}, \beta_{2}} t_{n}^{-\beta_{1}+\beta_{2}} .
$$

see [11]. Using Lemma 3.6 we get

$$
\begin{aligned}
\left\|\theta_{n}\right\| \leq & \left\|\rho_{n_{2}}\right\|+C \tau \sum_{j=1}^{n_{2}} t_{n-j+1}^{-1}\left\|\rho_{j}\right\|+C \tau \sum_{j=n_{2}+1}^{n}\left\|\bar{\partial}_{t} \rho_{k, j}\right\| \\
& +C \tau \sum_{j=1}^{n} t_{n-j+1}^{-1 / 2}\left\|f\left(U_{k, j-1}^{\mathrm{ms}}\right)-f\left(U_{j-1}\right)\right\|_{H^{-1}}
\end{aligned}
$$

and together with Lemmas 3.5 and 5.2 this gives

$$
\begin{aligned}
\left\|\theta_{n}\right\| \leq & C\left(H+k^{d / 2} \mu^{k}\right)^{2}\left(t_{n_{2}}^{-1 / 2}+\tau \sum_{j=1}^{n_{2}} t_{n-j+1}^{-1} t_{j}^{-1 / 2}+\tau \sum_{j=n_{2}+1}^{n} t_{j}^{-3 / 2}\right) \\
& +C \tau \sum_{j=1}^{n} t_{n-j+1}^{-1 / 2}\left\|U_{k, j-1}^{\mathrm{ms}}-U_{j-1}\right\| \\
\leq & C\left(H+k^{d / 2} \mu^{k}\right)^{2} t_{n}^{-1 / 2}+C \tau \sum_{j=1}^{n} t_{n-j+1}^{-1 / 2}\left\|e_{j-1}\right\| .
\end{aligned}
$$

Now consider $\theta_{1}$. We can rewrite

$$
\begin{aligned}
\theta_{1} & =E_{k, 1}^{\mathrm{ms}} \theta_{0}+\tau E_{k, 1}^{\mathrm{ms}} P_{k}^{\mathrm{ms}}\left(f\left(U_{k, 0}^{\mathrm{ms}}\right)-f\left(U_{0}\right)-\bar{\partial}_{t} \rho_{1}\right) \\
& =E_{k, 1}^{\mathrm{ms}} P_{k}^{\mathrm{ms}} e_{0}-E_{k, 1}^{\mathrm{ms}} P_{k}^{\mathrm{ms}} \rho_{1}+\tau E_{k, 1}^{\mathrm{ms}} P_{k}^{\mathrm{ms}}\left(f\left(U_{k, 0}^{\mathrm{ms}}\right)-f\left(U_{0}\right)\right),
\end{aligned}
$$


and using similar arguments as above

$$
\left\|\theta_{1}\right\| \leq C\left(H+k^{d / 2} \mu^{k}\right)^{2} t_{1}^{-1 / 2}+\tau t_{1}^{-1 / 2}\left\|e_{0}\right\|,
$$

Hence, we arrive at the estimate

$$
\left\|e_{n}\right\| \leq C t_{n}^{-1 / 2}\left(H+k^{d / 2} \mu^{k}\right)^{2}+C \tau \sum_{j=1}^{n} t_{n-j+1}^{-1 / 2}\left\|e_{j-1}\right\|, \quad n \geq 1
$$

and we can use Lemma 5.5 to conclude (5.11).

\section{Numerical results}

In this section we present two numerical examples to verify the predicted error estimates presented for the linear problem in Sect. 4 and the semilinear problem in Sect. 5. In both cases the domain is set to the unit square $\Omega=[0,1] \times[0,1]$ and $T=1$. The domain $\Omega$ is discretized with a uniform triangulation and the interval $[0, T]$ is divided into subintervals of equal length.

The method is tested on two different problems. One with constant coefficients

$$
c_{1}(x)=1, \quad A_{1}(x)=\left(\begin{array}{ll}
1 & 0 \\
0 & 1
\end{array}\right)
$$

and one with multiscale coefficients

$$
c_{2}(x)=D(x), \quad A_{2}(x)=\left(\begin{array}{cc}
B(x) & 0 \\
0 & B(x)
\end{array}\right),
$$

where $B$ and $D$ are piecewise constant with respect to a uniform Cartesian grid of size $2^{-6}$, see Fig. 1 for a plot of a typical coefficient. This choice of $B$ and $D$ imposes significant multiscale behavior on the coefficients. We expect quadratic convergence in the space of classical finite element with piecewise linear and continuous polynomials (P1-FEM) when $A=A_{1}$ and $c=c_{1}$, but poor convergence when $A=A_{2}$ and $c=c_{2}$. For the GFEM we expect quadratic convergence in both cases. Note that in the semilinear case we have $c=c_{1}$ in both examples.

We compute the localized GFEM in (3.16) and (5.10), denoted $U_{k, n}^{\mathrm{ms}}$, for 5 different values of the coarse grid width, $H=\sqrt{2} \cdot 2^{-2}, \sqrt{2} \cdot 2^{-3}, \sqrt{2} \cdot 2^{-4}, \sqrt{2} \cdot 2^{-5}$, and $\sqrt{2} \cdot 2^{-6}$. The time step is chosen to $\tau=0.01$ for all problems. The reference mesh $\mathcal{T}_{h}$ is of size $h=\sqrt{2} \cdot 2^{-7}$ and defines the space $V_{h}$ on which the localized corrector problems $\phi_{k, x}$ are solved. To measure the error, the solution $U_{n}$ in (3.3) is computed using P1-FEM on the finest scale $h=\sqrt{2} \cdot 2^{-7}$ with $\tau=0.01$.

Note that this experiment measures the error $\left\|U_{n}-U_{k, n}^{\mathrm{ms}}\right\|$. The total error $\| u\left(t_{n}\right)-$ $U_{k, n}^{\mathrm{ms}} \|$ is also affected by the difference $\left\|u\left(t_{n}\right)-U_{n}\right\|$, which is dominating for the smaller values of $H$. We now present the results in two separate sections. 


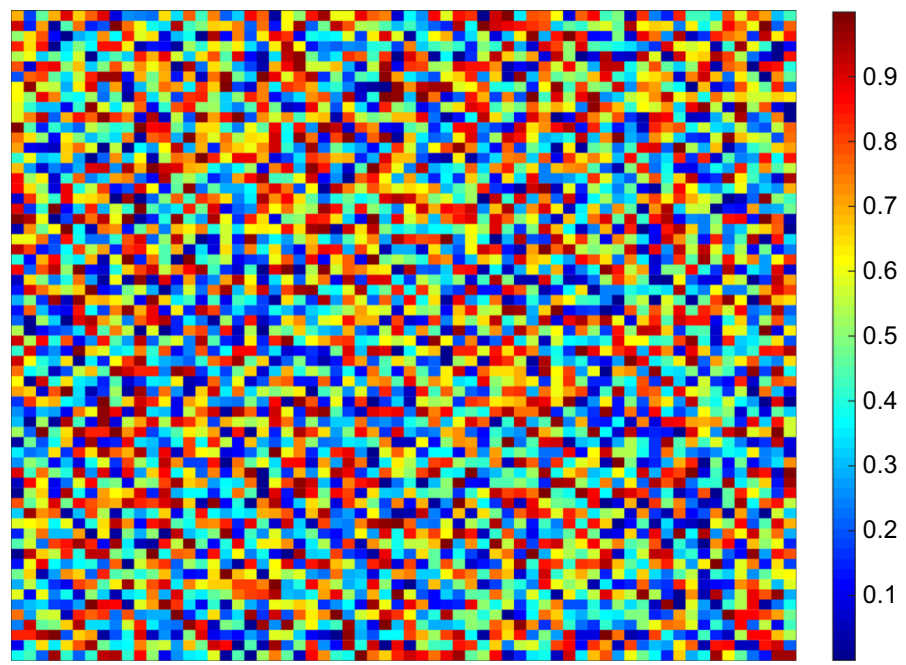

Fig. 1 A plot of a coefficient that is piecewise constant on a Cartesian grid of size $2^{-6}$

\subsection{Linear parabolic problem}

For the linear parabolic problem (2.1) the right hand side is set to $f(x, t)=t$, which fulfills the assumptions for the required regularity. For simplicity the initial data is set to $u_{0}=1$. To construct $B$ and $D$ we choose, for each cell in the Cartesian grid, a value from the interval $\left[10^{-1}, 10^{3}\right]$. Note that we choose different values for $B$ and $D$. This procedure gives both $B$ and $D$ rapidly varying features, see Fig. 1.

For each value of $H$ the localized GFEM, $U_{k, n}^{\mathrm{ms}}$, and the corresponding P1-FEM, denoted $U_{H, n}$, are computed. The patch sizes $k$ are chosen such that $k \sim \log \left(H^{-1}\right)$, that is $k=1,2,2,3$, and 4 , for the five simulations. When computing $U_{H, n}$ the stiffness matrix is assembled on the fine scale $h$ and then interpolated to the coarser scale. This way we avoid quadrature errors. The convergence results for the two examples are presented in Fig. 2, where the error at the final time $t_{N}$ is plotted against the degrees of freedom $|\mathcal{N}|$. Comparing the plots we can see the predicted quadratic convergence for the localized GFEM. Note that even though the multiscale features of $c$ are not included in the construction of the multiscale space we get convergence without preasymptotic effects, as suggested by the theory. However, as expected, the P1-FEM shows poor convergence on the coarse grids when the coefficients have multiscale features. We clearly see the pre-asymptotic effects when $H$ does not resolve the fine structure of $B$.

\subsection{Semilinear parabolic problem}

For the semilinear problem we study the Allen-Cahn equation, which has right hand side $f(u)=-\left(u^{3}-u\right)$ that fulfills the necessary assumptions. We define the initial data to be $u_{0}(x, y)=x(1-x) y(1-y)$, which is zero on $\partial \Omega$. The matrix $B$ constructed as 
(a)

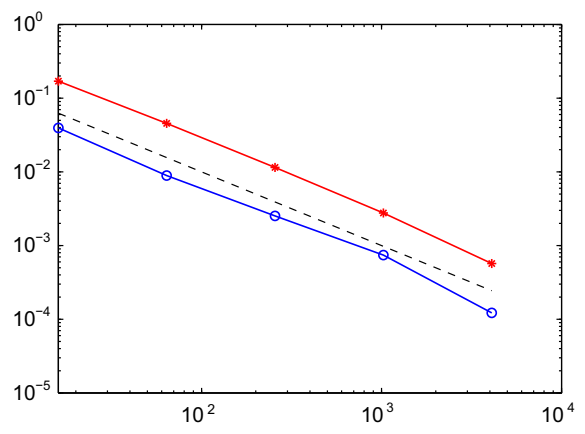

(b)

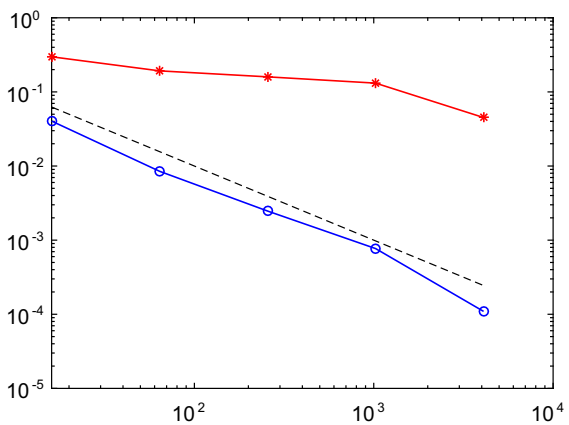

Fig. 2 Relative $L_{2}$ errors $\left\|U_{k, N}^{\mathrm{ms}}-U_{h, N}\right\| /\left\|U_{h, N}\right\|$ (blue circle) and $\left\|U_{H, N}-U_{h, N}\right\| /\left\|U_{h, N}\right\|$ (red asterisk) for the linear parabolic problem plotted against the number of degrees of freedom $|\mathcal{N}| \approx H^{-2}$. The dashed line is $H^{2}$. a Constant coefficients $c_{1}$ and $A_{1}$. b Multiscale coefficients $c_{2}$ and $A_{2}$ (color figure online)

(a)

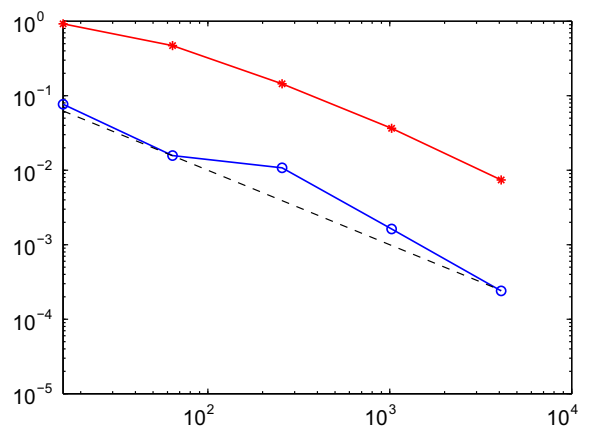

(b)

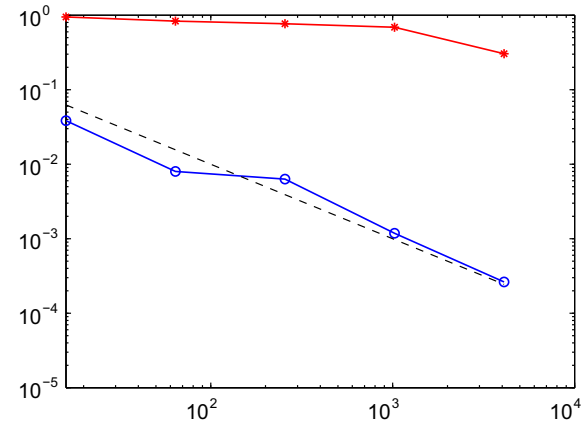

Fig. 3 Relative $L_{2}$ errors $\left\|U_{k, N}^{\mathrm{ms}}-U_{h, N}\right\| /\left\|U_{h, N}\right\|$ (blue circle) and $\left\|U_{H, N}-U_{h, N}\right\| /\left\|U_{h, N}\right\|$ (red asterisk) for the semilinear parabolic problem plotted against the number of degrees of freedom $|\mathcal{N}| \approx H^{-2}$. The dashed line is $H^{2}$. a Constant coefficient $A_{1}$. b Multiscale coefficient $A_{2}$ (color figure online)

in the linear case but with values varying between $10^{-3}$ and 1 . Note that, for simplicity, we have $c=1$ in both cases.

As in the linear case, we now compute the localized GFEM approximations $U_{k, n}^{\mathrm{ms}}$ and the corresponding P1-FEM, $U_{H, n}$. The patch sizes are chosen to $k=1,2,2,3$, and 4 , for the five simulations. The convergence results for the two examples are presented in Fig. 3. We can draw the same conclusions as in the linear case. The localized GFEM shows predicted quadratic convergence in both cases, but P1-FEM shows poor convergence on the coarse grids when the coefficients have multiscale features.

Open Access This article is distributed under the terms of the Creative Commons Attribution 4.0 International License (http://creativecommons.org/licenses/by/4.0/), which permits unrestricted use, distribution, 
and reproduction in any medium, provided you give appropriate credit to the original author(s) and the source, provide a link to the Creative Commons license, and indicate if changes were made.

\section{References}

1. Abdulle, A., Henning, P.: Localized orthogonal decomposition method for the wave equation with a continuum of scales. Math. Comput. 86(304), 549-587 (2017)

2. Babuska, I., Lipton, R.: Optimal local approximation spaces for generalized finite element methods with application to multiscale problems. Multiscale Model. Simul. 9(1), 373-406 (2011)

3. Bank, R.E., Yserentant, H.: On the $H^{1}$-stability of the $L_{2}$-projection onto finite element spaces. Numer. Math. 126(2), 361-381 (2014)

4. Carstensen, C., Verfürth, R.: Edge residuals dominate a posteriori error estimates for low order finite element methods. SIAM J. Numer. Anal. 36(5), 1571-1587 (1999)

5. Evans, L.C.: Partial Differential Equations. Graduate Studies in Mathematics, vol. 19, 2nd edn. American Mathematical Society, Providence (2010)

6. Henning, P., Målqvist, A.: Localized orthogonal decomposition techniques for boundary value problems. SIAM J. Sci. Comput. 36(4), A1609-A1634 (2014)

7. Henning, P., Målqvist, A., Peterseim, D.: A localized orthogonal decomposition method for semi-linear elliptic problems. ESAIM Math. Model. Numer. Anal. 48(5), 1331-1349 (2014)

8. Hughes, T.J.R., Feijóo, G.R., Mazzei, L., Quincy, J.B.: The variational multiscale method-a paradigm for computational mechanics. Comput. Methods Appl. Mech. Eng. 166(1-2), 3-24 (1998)

9. Ladyženskaja, O.A., Solonnikov, V.A., Uralceva, N.N.: Linear and Quasilinear Equations of Parabolic Type. Translated from the Russian by S. Smith. Translations of Mathematical Monographs, vol. 23. American Mathematical Society, Providence (1968)

10. Larson, M.G., Målqvist, A.: Adaptive variational multiscale methods based on a posteriori error estimation: energy norm estimates for elliptic problems. Comput. Methods Appl. Mech. Eng. 196(21-24), 2313-2324 (2007)

11. Larsson, S.: Nonsmooth Data Error Estimates with Applications to the Study of the Long-Time Behavior of Finite Element Solutions of Semilinear Parabolic Problems. preprint 1992-3. Department of Mathematics, Chalmers University of Technology (1992)

12. Larsson, S.: Semilinear parabolic partial differential equations: theory, approximation, and application. In: New Trends in the Mathematical and Computer Sciences. International Conference on Mathematics and Computer Science (ICMCS), vol. 3, pp. 153-194. Publications of the ICMCS, Lagos (2006)

13. Målqvist, A., Peterseim, D.: Localization of elliptic multiscale problems. Math. Comput. 83(290), 2583-2603 (2014)

14. Målqvist, A., Peterseim, D.: Computation of eigenvalues by numerical upscaling. Numer. Math. 130(2), 337-361 (2015)

15. Owhadi, H., Zhang, L.: Homogenization of parabolic equations with a continuum of space and time scales. SIAM J. Numer. Anal. 46(1), 1-36 (2007/08)

16. Owhadi, H., Zhang, L., Berlyand, L.: Polyharmonic homogenization, rough polyharmonic splines and sparse super-localization. ESAIM Math. Model. Numer. Anal. 48(2), 517-552 (2014)

17. Peterseim, D.: Variational multiscale stabilization and the exponential decay of fine-scale correctors. In: Barrenechea, G.R., Brezzi, F., Cangiani, A, Georgoulis, E.H. (eds.) Building Bridges: Connections and Challenges in Modern Approaches to Numerical Partial Differential Equations. Lecture Notes in Computational Science and Engineering, vol. 114. Springer (2016). Also available as INS Preprint No. 1509

18. Thomée, V.: Galerkin Finite Element Methods for Parabolic Problems. Springer Series in Computational Mathematics, 2nd edn. Springer, Berlin (2006)

19. E, W., Engquist, B.: The heterogeneous multiscale methods. Commun. Math. Sci. 1(1), 87-132 (2003) 